\title{
Some Tonal and Rhythmical Sequences in the Vocal Language of Dogs as Significant Earthquake Precursors
}

\author{
Giovanna de Liso ${ }^{1,2,3}$ \\ "'Seismic Precursors Study Center” (SPSC), Via Servera, 16, Torre Pellice, Italy \\ ${ }^{2}$ Istituto di Alta formazione artistica e musicale "G. F. Ghedini”, Cuneo, Italy \\ ${ }^{3}$ Voce Pinerolese, P. S. Donato 30, Pinerolo, Italy \\ Email: giovannadelisomr53@yahoo.it, giovan na deliso@conserva torioghedini.it, direttore@vocepinerolese.it
}

How to cite this paper: de Liso, G. (2018) Some Tonal and Rhythmical Sequences in the Vocal Language of Dogs as Significant Earthquake Precursors. Open Journal of Earthquake Research, 7, 221-268. https://doi.org/10.4236/ojer.2018.74013

Received: September 14, 2018

Accepted: November 25, 2018

Published: November 28, 2018

Copyright $\odot 2018$ by author and Scientific Research Publishing Inc. This work is licensed under the Creative Commons Attribution International License (CC BY 4.0).

http://creativecommons.org/licenses/by/4.0/

(c) (i) Open Access

\begin{abstract}
A monitoring of multiple physical parameters in a moderate seismic area in Western Piedmont (NW Italy) and the simultaneous observation of the behaviour of numerous species of domestic and wild animals gave in a period of over twenty years the possibility to distinguish the unusual animal behaviours due to local earthquake nucleation from other causes. In particular, the observation of the body and vocal language of dogs (Canis familiaris) in the same area has permitted not only to specify the different meanings of vocal language in connection to their body language, but also to classify the minimum elements into a vocal language that is linked together by tonal and rhythmical sequences of sounds that form a semantic lexicon. The usage of the same tonal and rhythmical vocal sequences in similar or identical situations, which are experienced by different groups of dogs, induces us to verify whether it could be possible to link particular vocal sequences to precise physical anomalies before earthquakes. The individuation of physical anomalies due to an earthquake nucleation or due to a hydro-geological destabilization, is possible thanks to a continuous long-term monitoring of some parameters. Moreover, the complexity of the vocal language of dogs increases if the dogs live in an area with a law population density. Then the correlation between some vocal sequences and some seismic precursors is better if dogs live free in yard or on farms, if they are in good health, and if they can establish a strong social relation of group. When dogs live closed in yards of houses that are far apart, they communicate with each other with an amazing vocal language, full of questions and answers, imitations of sequences, and information about situations that may be harmful to them.
\end{abstract}

\section{Keywords}

Vocal Language, Dogs, Rhythmical And Tonal Sequences, Syntax, Formant, 
Vocal Tract, Semantics, Seismic Precursors, Earthquakes, Magnetic

Declination, Sudden Commencement, Infra-Sounds, Brontides

\section{Introduction}

Research about possible seismic precursors is conducted in the "Seismic Precursors Study Centre" (SPSC). This Centre is located in Torre Pellice $\left(44^{\circ} 49^{\prime} 235^{\prime \prime} \mathrm{N}\right.$, $7^{\circ} 123^{\prime} 04^{\prime \prime E}$, Western Piedmont, NW Italy) at $699 \mathrm{~m}$, above sea level on Vandalino Mountain [1]. This research is founded on two levels of contemporaneous observations: observations about eventual anomalies of some physical parameters, recorded in a multiple parameter monitoring, and observations on the behaviours of domestic and wild animals [2] [3] [4].

The daily monitoring allows the possibility to distinguish which values are regular and which represents anomalies, on the ground of comparison with mean values of physical parameters; the personal visual and auditory observations of animal behaviours, living in a radius of $250 \mathrm{~m}$, from S.P.S.C, and the collection of irregular reports of farmers living within in a radius of $5-10 \mathrm{~km}$ from the Centre, give us a good knowledge about a larger eco-system.

The good collaboration with some local farmers is also due to the fact that they have non-intensive cow or sheep breeding, so they can observe their few animals better. Then, their dogs are often sheep-dogs, setters, pointers or crossbreeds, so the necessity of the farmers and dogs working together raises the level of their understanding. This is very helpful to research, because these farmers have a daily rapport with their animals, so they can notice eventual unusual behaviour and tell to the Centre about them.

Through traditional narrations, many inhabitants of Val Pellice also remember that on April $2^{\text {nd }} 1808$, a big earthquake occurred, with a magnitude that was recently estimated to have been $\mathrm{ML}=5.7$ degrees [5], and before the seismic shock, causing anomalous animal behaviours and weather anomalies [6] [7], people were induced to go outside of their houses. It can be supposed that this historical event could give local people a greater awareness when a researcher asks them to collaborate.

The purpose of this research is to verify if dogs can in some way signal the earthquakes well in advance, not only with anomalous behaviours of fear or anxiety, but also with particular vocalizations that take on a semantic character. This possible ability of the dogs, which I will show in this article to be linked to physical anomalies of the magnetic field of the earth, in turn seismic precursors, is analyzed from the rhythmical and melodic point of view of those vocal sequences emitted only when the magnetic declination anomalies appear several hours before local earthquakes. Therefore the vocalisations and the anomalous behaviours that precede the earthquakes with a $40-70$ seconds time advance (phase C) [8] are not considered precursors of the earthquakes, since in this case it is the auditory perception of the $p$ waves by the dogs. 


\section{Dog Behaviour Study: Methodology and Classifications of Dog Communications}

During the long period of the local earthquake study, the comparison of physical and chemical anomalies with unusual animal behaviours shows that dogs are the animals that best announce that an earthquake is about to occur [9] [10] [11]. We can note this correlation also for local seismic events with a low magnitude, with $M L \leq 2$, this consideration is possible only in retrospect, when we can verify, thanks to the INGV catalogue, whether the foreseen earthquake has really occurred.

The aim of the seismic precursors study (if the precursors exist) is to improve the temporal and the spatial forecast of the earthquake, with instrumentation development, monitoring physical parameters more and more, and understanding animal language better, especially canine language.

A moderately seismic area can also show different precursors, some of them have a significance of specific precursors, so in the areas of Cuneo and Val Pellice (West Piedmont), radioactive and geo-magnetic variations are the best precursors.

For a good space-time earthquake forecast, we must consider local seismic history and the whole body of precursors, giving each precursor a statistical weight.

Sometimes a few of the precursors observed in a seismic area are not present before a seism, but up to now, unusual dog behaviours have always been observed before earthquakes, with forecast time that will be analysed in this relation.

1) The study of dog behaviour also concerns the observation of the different forms of communication. All vegetal and animal creatures have a system of communication, more or less complex, with others around them, for an indispensable vital exigency. This system can be based on electrical discharges, variations of colours, luminous communications, hormone secretions (pheromones), physiological excretions, different postures of body and movements.

These different forms belong to their non-verbal communication. Humans also have a complex non-verbal communication, which is often unconscious [12] [13].

In Table 1 we can see animal communications summarized.

A few of forms of non-verbal communication can be designed for others who are far away, such as pheromones, excretions... but the body language that concern postures and body movements is addressed to an eventual interlocutor that is close by or not too far away.

A few forms of non-verbal communication of animals are associated to reproductive needs, others to the individual's and species' defence or to a request for help.

Dogs have a lot of forms of non-verbal communication and body language, with postures and body movements, constituting a complex system for expressing their emotional state and their intentions towards each other. 
Table 1. Animal non-verbal communications.

\begin{tabular}{cc}
\hline \multicolumn{2}{c}{ Animal communications } \\
\hline Non-verbal communications & Examples \\
Electrical discharges & e.g. torpedo \\
Colour variations & e.g. chameleon \\
Luminous communication bioluminescence & e.g. some shellfish, firefly, plankton \\
Hormone secretions pheromones & Animal metabolism \\
Physiological excretions & Urine and feces \\
Body language: postures and movements & $\begin{array}{c}\text { Relax state, alert, curiosity, dominant aggression, } \\
\text { fearful aggression, stress, fear and worry, extreme } \\
\text { fear and total submission, playfulness, courtship }\end{array}$ \\
\hline
\end{tabular}

Dogs are animals with a strong ability of social relationship. Their social group can have codes of conduct similar to those that govern groups of wolves. A dog, that we call an "alpha dog" [14], imposes itself on the group, with the right to attack first if the enemy is near or to bark first with particular vocal sequences if the "danger" is far away.

This behaviour is very interesting with regard to the possibility that "alpha dog" could be a "lookout dog" for seismic risk. We must also consider that the different body postures of dogs depend on both the external situation, and on the sensitivity and character of each dog [15] [16].

The interaction between the external situation, the emotions inducted and character can result in the body postures summarized in Table 2.

\section{Animal Vocal Communications}

\subsection{Different Modalities}

Animal communication is a result of a long evolution of species. Generally, the complex synergy of sensory organs for each species potentiates the function which best solves the problems of survival. So the auditory communication can reach the recipients of the message also outside the visual range.

The terrestrial animals hold the primacy of the ability to emit sounds and noises compared to aquatic creatures. Even Insects can emit sounds: in the male cicadas, chirping is issued by denticulate skin formations located in their legs. Some Beetles vibrate a membrane.

But the evolutionary leap in the development of a complex language begins with the differentiation of the larynx (organ typical to Vertebrates) in Amphibians.

In the Anuros, two parallel creases at the edge of the glottis act as vocal cords, and are separated into the arytenoid and the cricoid cartilages. Reptiles also have a similar structure. Birds have a larynx, but phonation function depends on the "syrinx".

In Mammals, the vocal cords are amidst the arytenoid cartilages and the thyroid gland, but there are two new cartilages, thyroid and epiglottis: this anatomic 
Table 2. Dog postures.

\begin{tabular}{|c|c|}
\hline Characteristic reaction & Body postures of dogs \\
\hline Relaxed and approachable: the dog is content & $\begin{array}{l}\text { Ears up, head high, mouth slightly open, tongue } \\
\text { exposed, tail down and relaxed, weight on four feet }\end{array}$ \\
\hline Alert: dog is interested in something out there & $\begin{array}{l}\text { Eyes wide, ears forward to catch a sound, smooth } \\
\text { nose and forehead, Posture slightly forward, } \\
\text { horizontal tail that may move from side to side }\end{array}$ \\
\hline Dominant aggressive & $\begin{array}{l}\text { Forehead may have vertical wrinkles, nose } \\
\text { wrinkled, teeth are bared, and corner of mouth is } \\
\text { tightened, body forward, ears forward, hackles } \\
\text { raised, tail raised and bristling, and wagging from } \\
\text { side to side }\end{array}$ \\
\hline Fearful and aggressive & $\begin{array}{l}\text { Pupils dilated, ears back, nose wrinkled, lips } \\
\text { slightly curled, corner of mouth pulled back, } \\
\text { hackles raised, tail tucked, body lowered }\end{array}$ \\
\hline $\begin{array}{l}\text { Stressed and distressed: the dog is not } \\
\text { submissive and may attack }\end{array}$ & $\begin{array}{l}\text { Pupils dilated, ears back, nose wrinkled, corner of } \\
\text { mouth pulled back, tail down, body lowered }\end{array}$ \\
\hline Fearful and worried & $\begin{array}{l}\text { Smooth forehead, ears back, licks at face of } \\
\text { dominant dog, corner of mouth pulled back, paw } \\
\text { raised, tail down, body lowered, footprints }\end{array}$ \\
\hline Total submission & $\begin{array}{l}\text { Smooth nose and forehead, ears flat and back, eyes } \\
\text { partly closed, head turned to avoid direct eye } \\
\text { contact, tail tucked, exposition of stomach and } \\
\text { throat, a few drops of urine }\end{array}$ \\
\hline Playfulness & $\begin{array}{l}\text { Ears up, head high, mouth slightly open, tongue } \\
\text { exposed, pupils dilated, forepaws lowered }\end{array}$ \\
\hline
\end{tabular}

structure permits mammals to have a better condition for developing an increasingly articulated language.

Broca's area and Wernicke's area are lacking in the brains of apes: the lower space between the epiglottis and soft palate limits the emission of sounds and the reduced mobility of the tongue limits the articulation of sounds and the resonances. In human individuals, Broca's area governs the functions of language articulations, Wernicke's area governs the functions of regulation of the sequences of sounds emitted and of the comprehension of language. Then, in the human body, the position of the larynx is very low, between the fourth and sixth cervical vertebra, a position that determines a wider space between the vocal cords and mouth, so a voice sounding board is possible [17] [18]. Evolution of the human species has also selected a reduction of the jawbone, behind the incisors, and an increased mobility of the tongue.

The reduction of the jawbone involves a different position of the facial and head muscles. These elements gave an important contribution to the evolution of human language [19].

Vertebrate phonation is the process by which the vocal folds produce certain sounds through quasi-periodic vibration. It is the result of a complex synergy of inhalation and exhalation of muscles and cartilages of the larynx, and of the ab- 
dominal muscles for the sound support and of numerous rear lateral trunk muscles for the "appoggio" of the sound, for the dynamic of vocal communications and sound quality.

The "appoggio" is given by the expiratory control, through which the subject slows the ascent of the diaphragm, maintaining the contraction of the external inter-costal muscles and the upper rear toothed muscle. Sound support and the simultaneous "appoggio of voice" are a professional technique for singers and actors, aimed at an artistic controlling of spoken and sung phrases [20] [21]. So, this technique is learned, but a simultaneous synergy between two muscular systems in phonation is also instinctively created when certain situations request a high intensity of the sounds of the communication.

Obviously, this last vocal modality only has the finality of counteracting the reduction of sound intensity in the space. This is very evident in dog, cat, cow, sheep, and horse communications. This complexity forms a strength and intrinsic relation between the emotional state and an unavoidable communication.

The characteristic of the sound is also connected to the geometric structure of the body, to the position of the larynx, to the length of vocal cords, to vocal tract length, to the distance between the temporo-mandibular joints, to the width of the cranium and so on.

Then, during the phonation, the coordination of the various muscles also depends on body posture, on walking upright or on all four legs, and on the position of the pelvis. This is true for both humans and other animals.

Figure 1 shows Broca's and Wernike's areas in the human brain.

Figure 2 shows the human facial muscles and the skull, whose geometric structure reached in millennia of evolution has led man to develop an extremely complex and articulator vocal language, unlike the other Mammals, including the dog.

Figure 3 shows the laryngeal cartilages, present in the Mammals and false and true vocal cords.

\subsection{Characteristics of Dog Voice in Relation to Size, Breed, Width of Cranium, and Body Posture}

The long selection of dog breeds by man has created numerous sizes and varied the geometrical relationships of the different parts of the skeletal system.

Similarly the characteristics of the human voice, according to a large size, (body weight and height of the legs) are associated to an increase of the average length of the vocal cords, of the tyro-arytenoids, arytenoids, cricothyroid, and lateral cricoarytenoid muscles. These muscles are the intrinsic muscles of the larynx and they adjust the pitch, during the phonation.

So dogs of big size can emit sounds with lower frequencies, while dogs of small size produce higher frequencies. Vocal frequencies of the dogs can have an interval between $50-658 \mathrm{~Hz}$ and mostly low frequencies are emitted during growling. Also for dogs, we can split the timbre of voices into low, medium, and high. We note a different phonation of vocal timbre and extension between 


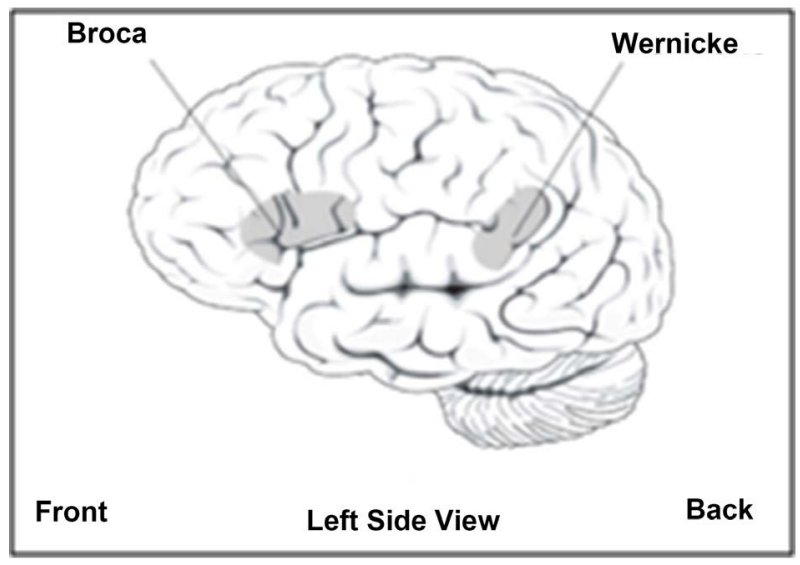

Figure 1. Broca's and Wernike's areas in the human brain. (Wikipedia, from "Wernike's area”).

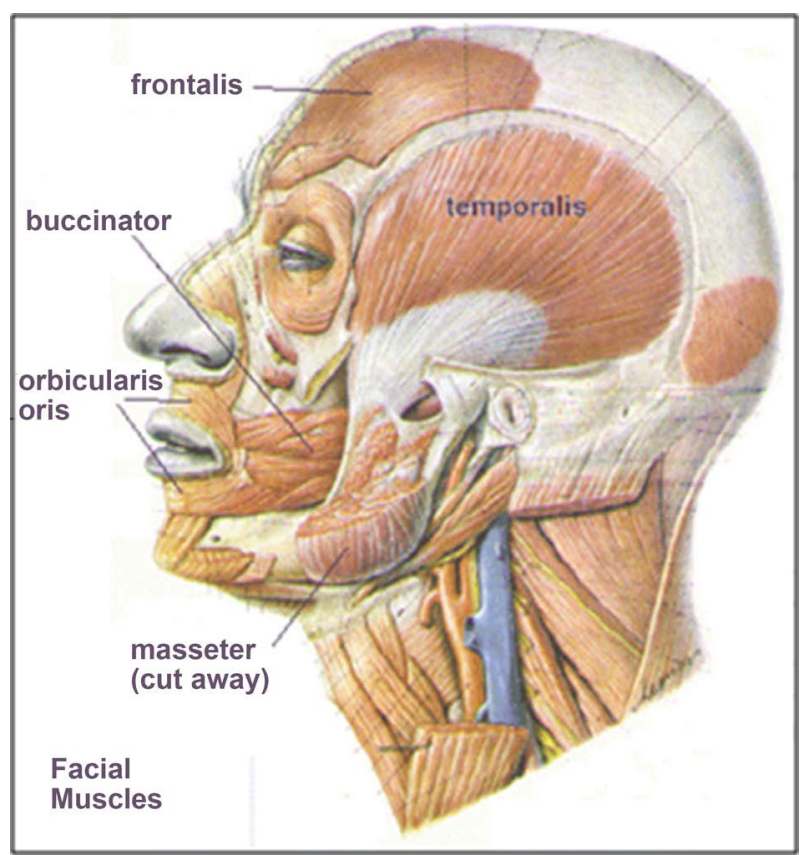

Figure 2. Facial muscles (Wikipedia from "facial muscles").

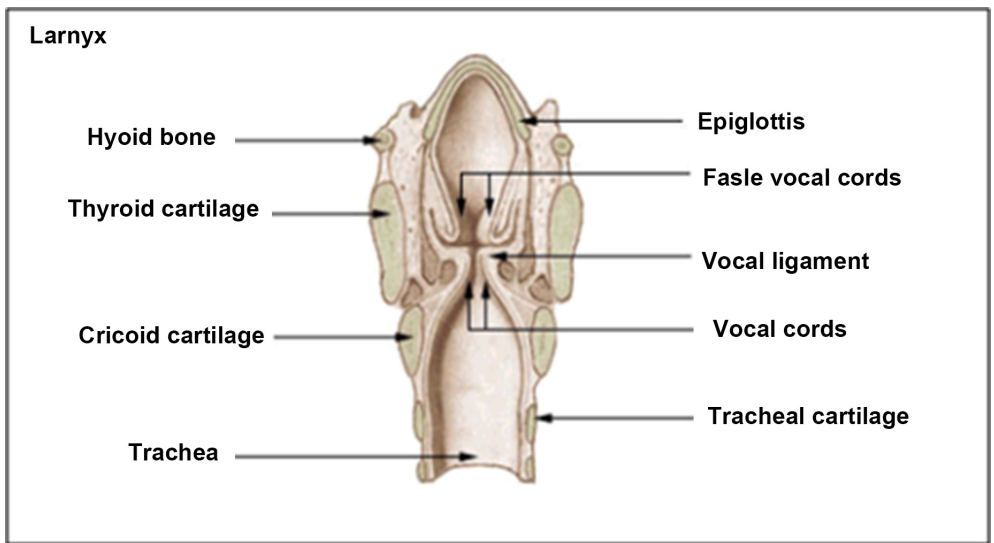

Figure 3. Larynx of mammals (Wikipedia, from "Larinx"). 
puppy and adult dogs. The differentiations between male and female voices of the adult are much smaller than we can observe in the context of sound frequency for human species.

In human species the larynx position of the newborn still six-seven months is very high. During breathing the soft palate and the epiglottis are in contact, the tongue is advanced and above the gum. When the newborn is sucking breast milk, the epiglottis is lifted and divides into two channels the isthmus of the jaws. This will allow baby breath and shallow at the same time. This is not possible for a human adult. This anatomy of breathing system of human babies is almost similar to those of the other Mammals, both puppies, both adults. Indeed, the other Mammals must breathe and shallow at the same time, because they must have always on nasal breathing for the defense. When the babies larynx alights and distances to soft palate, also the tongue modifies his position in relation to the jaws and it goes back. This new situation gives to human phonation a great possibility of articulations, this characteristic is only for human species.

Also for puppies there is a phase of hormonal changes, both for male, both for female, during which they pass from puberty to adolescence, similar to what happens for human adolescents. This period of dogs can last from six to fourteen months and it also involves psychological changes as well as physical changes, including variations in phonation.

Human sexual dimorphism poses considerable difficulty for the adolescent during this pubertal and post-pubertal phase. This "mute of the voice", which is found in a minority for the girls, implies a considerable difficulty in the laryngeal muscles due to the rapid lengthening of the vocal cords, so the male teenager easily passes from a register of childish voice to an adult of lower register. This is not so evident in dogs, for whom the difference between the extension of sounds between male adolescents and adults is not so remarkable.

Furthermore, the differentiations between male and female voices of the adult are much smaller that we can observe in the context of sound frequency for human species.

The vocal range and the tone of voice of dogs differ in the adult for the size of the dog, given by the breed and not for the sex.

The distance between the temporo-mandibular joints and the width of cranium are important parameters for the geometric structure that influences the formation of resonators during phonation: the pharynx, the oral and the nasal cavities. Then the modification of the soft palate (very long for dogs) during the phonation can modify in the vocal tract the characteristic of sound emitted (the vocal formants) and the quality of communication.

The evaluation of the morpho-types of dogs as the cephalic index (the ratio between the width, side to side, and length, front to back, of the cranium, skull) and the craniofacial ratio (length of cranium-muzzle) is interesting for understanding vocal language from the point of view of phonation physiology [22] [23]. 
On the other hand, the synergy between all sensorial organs gives the vocal communication a strength link with the psychological and ethological situation.

We can give a few examples.

The canine sense of smell is very developed, there are 120 - 300 millions of smell receptors. Dog's sense of smell is the first sense in terms of superior ability to discern the quality of odours and their persistence in the environment.

If a dog feels some animal intruder by smell, but cannot see them (example 1) and the smell of intruder is new, a quizzical reaction follows: there can be anxiety, curiosity, and aggressiveness, but in every emotional situation, the dog raises its head a little, so it can sniff the air with a greater volume. This position of the head implies the action of cricothyroid and cricoarytenoid muscles, over all the posterior cricoarytenoid muscle, which lengthens the vocal cords. This anatomic situation favours a vocal emission of high sound.

An interesting research by Liancai $\mathrm{Mu}$ and Shilin Yang [24] experimentally confirms with electromyography that during the dog's phonation, the posterior cricoarytenoid muscles are also contracted. So, in example 1, dogs often emit vocal sequences with two sounds; if $v_{1}$ is the frequency of the fundamental of the first sound emitted and $v_{2}$ of the second, we can have:

$$
\text { a] } \rightarrow v_{2}=3 / 2 v_{1}
$$

The frequency $v_{1}$ depends on the breed and on the agitation of the dog. The ratio

$$
\text { b] } \rightarrow v_{2} / v_{1}=3 / 2 \rightarrow \text { a perfect musical fifth }
$$

In this case the two sounds are emitted with discrete modality, with a breath between the two sounds or with a short break time.

If the aggression is too strong, (example 2) the time of transitional attack of the first sound is very short. The phonation energy is extremely intense and so it produces the sound that has a ratio with the first sound of a musical perfect fifth, and the body posture is of a dominant aggressive dog. In this case, between $v_{1}$ and $v_{2}$ there are numerous other frequencies and the higher sound is reached as the musical "glissando", or vocal "portamento". Different vocal sequences will be analysed in Chapter 4.

The lateral position of dog's eyes gives him a larger eyesight corner than human eyesight, this is aimed at the vision of the movements of possible enemies. The frontal eyesight is less acute, so dogs compensate this with their other senses.

The dog's hearing sense is the second to smelling sense in terms of discerning ability. The range of sound frequencies that humans can detect is including approximately between 20 - 12,000 - 20,000 hertz, depending on age. Dogs can hear the infra-sounds with lower limit of 3.5 - 7 hertz, while the upper limit can reach 40 -60,000 hertz, also depending on age. This ability of sensory organs will give in chapters 5, 6, 7 an explanation to unusual dog behaviors before earthquakes. 


\subsection{Group Dynamics}

Both non-verbal communications and both vocal-communications of dogs and wolves are strictly regulated by relationship rules in a group, with a rigid hierarchical scheme. The first rule, the leader rule, is won through struggles by group male members, the winner becomes the "alpha wolf" or the "alpha dog", it has the privilege of eating first, of going ahead, of reproducing, of howling (or barking for dogs) first. However, it must always defend its role and the welfare of the group with a constant level of attention, with an intense stress [25] [26].

When a dog enters a human family, a group is formed.

I have observed the following groups constituted thus:

1) an owner and one dog, that consider its owner as an "alpha dog",

2) an owner and one dog, that consider itself as an "alpha dog", due to a wrong course of the owner,

3) an owner, more human members of family and a dog, with owner as "alpha dog", the dog is normally the last in the hierarchy,

4) an owner and many dogs, the owner is the "alpha dog", in the group of dogs, a male dog becomes an "alpha dog" number two, so the hierarchy is constituted by human alpha 1 , dog alpha 2 , male dogs, female dogs,

5) an owner and many female dogs, also in the female group of dogs, there is an "alpha $\operatorname{dog} 2$ " subjected to owner, often the "alpha dog 2" mimics the male sexual act on female dogs of the group.

Furthermore, there are dog groups without owners. They are stray dogs and the group is strictly regulated by relationship rules similar to a wolf group. I have collected some testimonies from people living in the Cuneo area.

Sometimes, the group is not composed of stray dogs, but of dogs that have owners neglecting them. In 1995 in Torre Pellice, the dog Lulù (number 2 in Table 4), was seen many times by people while it was walking as an alpha dog of a group of four dogs or asking for food at the town's butchers. For this purpose, he knocked on the windows of the shops with his paw. One day I met him, I gave him food and cuddles and I adopted him, so I become his alpha dog-owner.

However Lulù never forgot his female mate (female alpha dog), because many times he ran out of my house to meet his female. One day Lulù ran out of the house and people told me that he watched over his mate for several hours, on the road, while his mate was agonizingly hit by a car. Lulù came home the next day and he refused food for some days. Then he did not run any more. This case shows that the relations of dogs with a group can be very strong, even more than its relations with its owners. But the relation of Lulù with his female mate also shows a capacity of intense emotional relationship and not just of dominance.

If a group is formed by wild dogs, it behaves as a wolf group, but it does not fear human species as wolfs do. It is a group swaggering towards humans.

A few shepherds told me about a sighting of a similar group on high mountain pastures.

In this research, a domestic dog was observed, the "Canis familiaris", that is a 
mammal of the "Canidi" family and Canis gender. With a phylogenetic analysis of ancestral relationships, the observations of group behaviors of dogs similar to those of a wolf group lead us to think that the Canis familiaris could have wolf origins. This hypothesis has been confirmed by recent studies on sequences of mtDNA mitochondrial.

Some researchers think that a few dog breeds could have been derived from jackals or coyotes, while the dog breeds of the North could have been derived from wolves, during the long process of breed selections by human, for the domestication of the primitive dog. But mtDNA mitochondrial sequences of dogs differ from those of wolves for $2 \%$, while from those from coyotes for $7.5 \%$. For a better understanding of the herd behaviour of dogs, we must also consider the relation between the character of every dog (due to the education received from owner and from its life situation), and it's belonging to a certain breed. It is important to consider four modes of behaviours of dogs in relation to an ancestral memory of four developmental stages of wolves, as shown in Table 3. It is interesting to note that belonging to one group more than to another for dog breeds is due to the morphology of the dog that recalls that of the growth stage of the wolf [27].

Table 4 shows number of dogs personally observed, or observed by their owners, dog breeds and distance between dog's house and SPSC are added.

The dogs 1, 2, 3, 4, 5, 6 and 7 are the dogs of the Author, with the exception of the dog No. 5, who was born at home, all the others were adopted.

Table 5 shows the number of dogs personally observed, their sex, breed, size, width of cranium and voice tone.

Table 6 shows the continuity of the observations by the author of the behaviour of his dogs, even in their alternation, the partial observations referred to the author by the other owners of the dogs considered and the partial ones made by the author regarding the other dogs.

\section{Language Structural Analysis}

The long period of observations on animal behaviours showed me a complex system of vocal communications in Mammals, especially in dogs, combined with a great ability for both individual and group strategies to resolve their numerous life problems. The ability to communicate an action project involves the ability to put a concept in relation with the corresponding vocalization or body posture, that assume a function of a signifier of that concept. Is it possible to apply the same language structuralism criteria of human language to dog language?

The answer to this question is very important in order to better understand dog language and to understand eventual precursor signals given by dogs before earthquakes or geological destabilizations.

My long listening experience has led me to identify, using both a frequency detector and the musician's analysis requirements, the recurrence of vocal structures, with frequencies of sounds and rhythmic modules identical over 
Table 3. Behaviours of dogs in relation to developmental stages of wolf and dogs breeds.

\begin{tabular}{|c|c|c|c|}
\hline Behaviour of dog & $\begin{array}{l}\text { Development } \\
\text { stage of wolf }\end{array}$ & Breeds of dogs & Character of dogs \\
\hline Neoteny & Puppy stage & $\begin{array}{c}\text { Molossians } \\
\text { macrocephalies }\end{array}$ & $\begin{array}{l}\text { Fighting dogs uncontrolled } \\
\text { aggression lack of curiosity }\end{array}$ \\
\hline Play & Wolf cub & $\begin{array}{l}\text { Hunting and } \\
\text { retrievers dogs }\end{array}$ & Playful and curious dogs \\
\hline Gregariousness & Youth & $\begin{array}{l}\text { Herding dogs run, border } \\
\text { collie, normo and } \\
\text { dolichocephalic dogs }\end{array}$ & $\begin{array}{l}\text { Gregarious and } \\
\text { cooperative dogs }\end{array}$ \\
\hline Hooker phase & Adult phase & $\begin{array}{l}\text { North and primitive dogs, } \\
\text { husky, samojied }\end{array}$ & Predatory dogs \\
\hline
\end{tabular}

Table 4. List of dogs observed.

\begin{tabular}{|c|c|c|c|}
\hline & Breeds & Distances in meter \\
\hline $\begin{array}{l}\text { Dogs and sex } \\
\text { 1) Priscilla }\end{array}$ & $\mathrm{f}$ & Dachshund & $\mathrm{d}=0$ \\
\hline 2) Lulù & $\mathrm{m}$ & Pomeranian Dog & $\mathrm{d}=0$ \\
\hline 3) Techila & $\mathrm{f}$ & $\begin{array}{l}\text { Italian Black Wolf: Wild Wolf } \\
\text { Mother/German Shepherd Father }\end{array}$ & $\mathrm{d}=0$ \\
\hline 4) Medea & $\mathrm{f}$ & German Shepherd & $\mathrm{d}=0$ \\
\hline 5) Anacleto & $\mathrm{m}$ & German Shepherd & $\mathrm{d}=0$ \\
\hline 6) Bianca & $\mathrm{f}$ & White Swiss Shepherd & $\mathrm{d}=0$ \\
\hline 7) Betlemma & $\mathrm{f}$ & Canaan Dog & $\mathrm{d}=0$ \\
\hline 8) Billy n. 1 & $\mathrm{~m}$ & Yorkshire Terrier & $10<\mathrm{d} \leq 50$ \\
\hline 9) // & $\mathrm{f}$ & Mongrel Poodle & $10<\mathrm{d} \leq 50$ \\
\hline 10) // & $\mathrm{m}$ & Mongrel Beagle & $10<\mathrm{d} \leq 50$ \\
\hline 11) // & $\mathrm{m}$ & Beagle & $10<\mathrm{d} \leq 50$ \\
\hline 12) // & $\mathrm{m}$ & German Shepherd & $50<\mathrm{d} \leq 80$ \\
\hline 13) Balù & $\mathrm{m}$ & German Shepherd & $50<\mathrm{d} \leq 80$ \\
\hline 14) Pachito & $\mathrm{m}$ & German Shepherd & $50<\mathrm{d} \leq 80$ \\
\hline 15 Techila & $\mathrm{f}$ & German Shepherd & $50<\mathrm{d} \leq 80$ \\
\hline 16) Rocky & $\mathrm{m}$ & Mongrel German Shepherd & $80<\mathrm{d} \leq 130$ \\
\hline 17) Argo & $\mathrm{m}$ & Mongrel German Shepherd & $80<\mathrm{d} \leq 130$ \\
\hline 18) Lilly & $\mathrm{f}$ & Mongrel Beagle & $80<\mathrm{d} \leq 130$ \\
\hline 19) // & $\mathrm{m}$ & Mongrel Beagle & $80<\mathrm{d} \leq 130$ \\
\hline 20) Cucciolo & $\mathrm{m}$ & Pit-Bull & $130<\mathrm{d} \leq 200$ \\
\hline 21) Billy n.2 & $\mathrm{m}$ & Yorkshire Terrier & $130<\mathrm{d} \leq 200$ \\
\hline 22) // f. & $\mathrm{f}$ & German Shepherd & $130<\mathrm{d} \leq 200$ \\
\hline 23) // & $\mathrm{f}$ & Jack Russell Terrier & $200<\mathrm{d} \leq 250$ \\
\hline 24) // & $\mathrm{f}$ & Jack Russell Terrier & $200<\mathrm{d} \leq 250$ \\
\hline 25) // & $\mathrm{m}$ & Jack Russell Terrier & $200<\mathrm{d} \leq 250$ \\
\hline 26) // & $\mathrm{m}$ & Jack Russell Terrier & $200<\mathrm{d} \leq 250$ \\
\hline 27) $\alpha$ dog of super group & $\mathrm{m}$ & German Shepherd & $200<\mathrm{d} \leq 250$ \\
\hline 28) // & $\mathrm{m}$ & Mongrel Poodle & $200<\mathrm{d} \leq 250$ \\
\hline 29) // & $\mathrm{f}$ & Mongrel Dachshund & $200<\mathrm{d} \leq 250$ \\
\hline 30) Trip & $\mathrm{m}$ & Mongrel Pit-Bull & $200<\mathrm{d} \leq 250$ \\
\hline
\end{tabular}


Table 5. List of dogs and their breed, size, cranium width and voice tone.

\begin{tabular}{|c|c|c|c|c|}
\hline $\begin{array}{l}\text { Dog } n . \\
\text { and sex }\end{array}$ & Breeds & Size & Cranium width & Voice tone \\
\hline 1) $f$. & Dachshund & small & $11.5 \mathrm{~cm}$. & medium \\
\hline 2) $\mathrm{m}$. & Pomeranian Dog & small & $11 \mathrm{~cm}$. & acute \\
\hline 3) $f$. & $\begin{array}{l}\text { Italian Black Wolf: Wild Wolf } \\
\text { Mother/German Shepherd Father }\end{array}$ & medium & $11.5 \mathrm{~cm}$ & medium \\
\hline 4) $\mathrm{f}$. & German Shepherd & medium & $12.5 \mathrm{~cm}$. & medium \\
\hline 5) $\mathrm{m}$ & German Shepherd & medium & $12.7 \mathrm{~cm}$ & low \\
\hline 6) $f$. & White Swiss Shepherd & medium & $11.5 \mathrm{~cm}$. & medium \\
\hline 7) $f$. & Canaan Dog & medium/large & $12 \mathrm{~cm}$. & low \\
\hline 8) $\mathrm{m}$. & Yorkshire Terrier & small & $10 \mathrm{~cm}$. & acute \\
\hline 9) $f$. & Mongrel Poodle & small & n.d & acute \\
\hline 10) $\mathrm{m}$. & Mongrel Beagle & medium & n.d & medium \\
\hline 11) $\mathrm{m}$. & Beagle & medium & $10 \mathrm{~cm}$ & low \\
\hline 12) $\mathrm{m}$. & German Shepherd & medium & n.d. & low \\
\hline 13) $\mathrm{m}$. & German Shepherd & medium & $12.5 \mathrm{~cm}$. & low \\
\hline 14) $\mathrm{m}$. & German Shepherd & medium & $12 \mathrm{~cm}$ & low \\
\hline 15) $f$. & German Shepherd & medium & n.d. & medium \\
\hline 16) $\mathrm{m}$. & Mongrel German Shepherd & medium/large & n.d & medium \\
\hline 17) $\mathrm{m}$. & Mongrel German Shepherd & medium/large & n.d & medium \\
\hline 18) $f$. & Mongrel Beagle & small & n.g & acute \\
\hline 19) $\mathrm{m}$. & Mongrel Beagle & small & n.d & acute \\
\hline 20) $\mathrm{m}$. & Pit-Bull & medium & n.d & medium \\
\hline 21) $\mathrm{m}$. & Yorkshire Terrier & small & n.d & acute \\
\hline 22) $f$. & German Shepherd & medium & n.d & medium \\
\hline 23) $f$. & Jack Russell Terrier & small & $9.3 \mathrm{~cm}$. & acute \\
\hline 24) $f$. & Jack Russell Terrier & small & $9.5 \mathrm{~cm}$. & acute \\
\hline 25) $\mathrm{m}$. & Jack Russell Terrier & small & $9.3 \mathrm{~cm}$. & acute \\
\hline 26) $\mathrm{m}$. & Jack Russell Terrier & small & $9.5 \mathrm{~cm}$. & acute \\
\hline 27) $\mathrm{m}$. & German Shepherd & medium & n.d & very low \\
\hline 28) $\mathrm{m}$. & Mongrel Poodle & small & n.d & acute \\
\hline 29) $\mathrm{f}$. & Mongrel Dachshund & small & n.d & acute \\
\hline 30) $\mathrm{m}$. & Mongrel Pit-Bull & small & $11 \mathrm{~cm}$. & acute \\
\hline
\end{tabular}

time, issued by the dogs under examination and by others outside the area of study, in similar situations of responses to the stresses of the environment.

The anatomy and the physiology of the voice of dogs do not allow a rich articulation of phonemes due to the shape of the muzzle and the lower mobility of the tongue, in relation to human vocal articulation ability. However, the millenary collaboration of dogs with human and their gregarious aspect with other dogs have gradually imposed upon dog language a greater complexity and diversification, both in increasingly ritualized body postures, and in increasingly 
Table 6. Observation modes.

\begin{tabular}{|c|c|c|c|c|}
\hline \multicolumn{2}{|c|}{ Dogs and sex } & \multirow{2}{*}{$\begin{array}{c}\begin{array}{c}\text { Period of personal } \\
\text { observations of } \\
\text { body/vocal language }\end{array} \\
1995-1996\end{array}$} & \multirow{2}{*}{$\begin{array}{c}\text { Period of personal } \\
\text { hearing observations of } \\
\text { vocal language }\end{array}$} & \multirow{2}{*}{$\begin{array}{c}\text { Period of data } \\
\text { collection of a few } \\
\text { observations by owners } \\
\text { of dogs } \\
-\end{array}$} \\
\hline 1) Priscilla & f. & & & \\
\hline 2) Lulù & $\mathrm{m}$. & $1995-2001$ & $1995-2001$ & - \\
\hline 3) Techila & f. & $1995-2001$ & $1995-2001$ & - \\
\hline 4) Medea & f. & 2001-2014 & 2001-2014 & - \\
\hline 5) Anacleto & $\mathrm{m}$ & 2001-2015 & 2001-2015 & - \\
\hline 6) Bianca & f. & 2014-2018 & 2014-2018 & - \\
\hline 7) Betlemma & f. & 2014-2018 & 2014-2018 & - \\
\hline 8) Billy n. 1 & $\mathrm{~m}$. & 2014-2018 & 2014-2018 & 2014-2018 \\
\hline 9) // & f. & - & 2013-2018 & - \\
\hline 10) // & m. & - & 2013-2018 & - \\
\hline 11) // & m. & - & $2012-2018$ & - \\
\hline 12) // & $\mathrm{m}$. & - & $2011-2018$ & - \\
\hline 13) Balù & $\mathrm{m}$. & - & 2014-2018 & 2014-2018 \\
\hline 14) Pachito & $\mathrm{m}$. & - & 2013-2018 & - \\
\hline 15) Techila & f. & & 2013-2018 & - \\
\hline 16) Rocky & $\mathrm{m}$. & - & $2012-2018$ & - \\
\hline 17) Argo & m. & - & 2012-2018 & - \\
\hline 18) Lilly & f. & - & $2012-2018$ & - \\
\hline 19) // & $\mathrm{m}$. & - & $2012-2018$ & - \\
\hline 20) Cucciolo & & - & 2014-2015 & 2014-2018 \\
\hline 21) Billy n.2. & $\mathrm{m}$ & - & 2014-2015 & \\
\hline 22) // & f. & - & 2014-2015 & \\
\hline 23) // & f & - & 2014-2015 & 2014-2018 \\
\hline $24 / /$ & & - & 2014-2015 & 2014-2018 \\
\hline 25) // & $f$ & - & 2014-2015 & 2014-2018 \\
\hline 26) // & $\mathrm{m}$ & - & 2014-2015 & 2014-2018 \\
\hline 27) // & $\mathrm{m}$ & - & 2011-2018 & 2011-2018 \\
\hline 28) // & $\mathrm{m}$ & - & 2011-2018 & 2011-2018 \\
\hline 29) // & f. & - & 2011-2018 & 2011-2018 \\
\hline 30) Trip & m. & - & 2017-2018 & 2017-2018 \\
\hline
\end{tabular}

structured vocal language. This can explain why the howling of wolves is more complex than that of dogs, and the barking of dogs is more articulated than that of wolves.

I can distinguish the different ways of dog vocalization, as shown in Table 7.

Table 8 instead shows various types of vocal expression not linked to semantic indications but to generic communications of emotional states, therefore not 
Table 7. Different ways of dog vocalizations.

\begin{tabular}{|c|c|c|}
\hline Vocalization & Emission of the sounds & Frequency of the sounds \\
\hline Howling & $\begin{array}{l}\text { Continuous sound emission like } \\
\text { singing, emitted alone or in group }\end{array}$ & $\begin{array}{l}\text { Medium-high frequencies of sounds } \\
\text { singing rich in various intonations in } \\
\text { extreme "legato" }\end{array}$ \\
\hline Whimpering/Moaning & Continuous sound emission & $\begin{array}{l}\text { High frequencies of sounds, with } \\
\text { numerous intervals of descendant } \\
\text { minor thirds }\end{array}$ \\
\hline Whining & $\begin{array}{l}\text { Continuous sound emission, similar } \\
\text { to a cried singing }\end{array}$ & $\begin{array}{l}\text { Medium-low frequencies of sounds, } \\
\text { with numerous descendant intervals } \\
\text { of a perfect musical fifth, in extreme } \\
\text { "legato" and with the transitional } \\
\text { attack release of the low sound that is } \\
\text { very short. The first sound is longer } \\
\text { than the last one, reached with a } \\
\text { glissando }\end{array}$ \\
\hline Growling & $\begin{array}{l}\text { Continuous sound emission due to } \\
\text { a short initial veil vibration, } \\
\text { followed by that of false vocal folds }\end{array}$ & Very low frequencies \\
\hline Barking & $\begin{array}{l}\text { Continuous or discrete emission of } \\
\text { short sounds }\end{array}$ & $\begin{array}{l}\text { Various numbers of frequencies, } \\
\text { emitted in significant tonal and } \\
\text { rhythmical sequences }\end{array}$ \\
\hline Yawning & $\begin{array}{l}\text { a) long inspiration without vocal } \\
\text { sounds } \\
\text { b) long inspiration followed by a } \\
\text { long exhalation with vocal sounds } \\
\text { on the same opening of the yawning }\end{array}$ & $\begin{array}{l}\text { a) no vocal sound } \\
\text { b) ascendant and descendant } \\
\text { intervals of open vowels }\end{array}$ \\
\hline Sighing & $\begin{array}{l}\text { Long inhalation and exhalation } \\
\text { without vocal sounds }\end{array}$ & // \\
\hline whistling & Continuous sound emission & High frequencies of sounds \\
\hline
\end{tabular}

Table 8. Different ways of dog vocalization in relation to emotional states.

\begin{tabular}{cl}
\hline Vocalization & \multicolumn{1}{c}{ Emotional states } \\
\hline Howling & $\begin{array}{l}\text { Social sharing of an emotion, strengthening of social ties if in a group, } \\
\text { state of melancholy and call for attention if in a state of solitude. } \\
\text { Request for attention by the puppy to the mother or the dog to the } \\
\text { owner, expression of physical or psychological pain, expression of } \\
\text { contentment at the arrival of the mother or the owner after a period of } \\
\text { shimpering/Moaning }\end{array}$ \\
Whining & $\begin{array}{l}\text { Intense physical pain, anguish of abandonment. } \\
\text { Growling }\end{array}$ \\
Intense aggression, intimidation signal to not get into a clash. \\
Sighing & $\begin{array}{l}\text { a) Paralinguistic signal to reduce stress } \\
\text { b) Contentment for having reduced stress }\end{array}$ \\
Anistling & It accompanies numerous other vocalizations.
\end{tabular}

semantic, analogously, especially with regard to the processing of dogs and wolves of the howl, to what happens in the a-semantic musical expressions.

This can make us understand why many animals, in particular dogs, are sensitive to music, especially to classical music, particularly to ancient music, whose 
structure is based on concatenations of the first natural harmonics of a sound.

Some dogs use the vocalization of pain in a dispute, out of fear, even if they have not been bitten in the slightest.

The happy experience of having closely observed wolves made me realize the similarity of the howling vocalizations of the groups of dogs and those of the wolves, while I did not have enough observation time to evaluate the quality of the barking of wolves.

Both the howling of the dogs and that of the wolves is configured as a highly creative song, full of alternating musical intervals of ascending fair fifths and minor thirds, major ascending sixths, followed by descendant major thirds, achieved with continuous "glissati" and on which there is a musical "crown", that is a lingering. Dogs and wolves begin the ululation preceded by a profound diaphragmatic inhalation, to manage the length of the phonation, then they lower their head, until reaching almost a line perpendicular to the ground with the lower jaw, while usually intoning a perfect fifth, many times.

They know how to perfectly modulate the pitch of the sounds and the dynamics of the phrase, working on the support of the voice. Moreover, they pass from a full register of voice, with complete vibration of the vocal cords, in the sense of the width, to one that we can define as "falsetto", using vocal musical jargon, that is to say, the vocal formants change in the vocal tract, modifying the glottal opening and the issuance of a " $u$ ". During this process they melodically create vocal ornaments like "mordants".

The howl of dogs can be triggered by the acoustic perception of the sirens of ambulances or the tolling of bells, probably due to the annoyance that these sounds arouse in them.

Some dogs howl when there is a sudden variation of terrestrial magnetic declination, at the same time that other dogs in the group emit precise sequences of barking. This form of discomfort of dogs can be considered a seismic precursor, since when I recorded instrumentally a variation of magnetic declination that occurred a few hours before a seismic event. This will be further analyzed in the following chapters.

Often it is the loneliness of a dog that induces it to unleash long sad howls and call for attention, in this case there are no answers with howling from other dogs.

If it is a male alpha wolf that howls due to sexual attraction, it can happen that other female dogs respond with howls. It happened a few times with my dog Techila, mother wolf daughter: sometimes the wolf came up to my house but there was a fence, so the wolf and my female dog could not have contacts except with their sense of smell. My female dog responded with thrilling moans, not like the other more distant dogs that reacted with aggressive barking. The wolf sometimes returned.

Before analyzing the vocal sequences of barking, it is useful to remember that, due to the anatomical conformation of the dog, few vowels and consonant are possible for the articulation of their language. 
We can distinguish a few vowels and consonants in dog vocal language, as shown in Tables 9-11.

We note that dogs emit posterior vowels, open or closed, increasing the angle of opening between the two jaws.

These diphthongs do not really consist of two vowels and therefore are not technically diphthongs.

It is better to consider them as sounds preceded by the semi-consonant $\mathrm{W}: \rightarrow \mathrm{Wa}^{*}, \mathrm{Wo}^{*}$.

The vestibular fold (ventricular fold, superior or false vocal cord) is one of two thick folds of mucous membrane, the vestibular ligament. This is attached in front to the angle of the thyroid cartilage, immediately below the attachment of the epiglottis, and behind, to the antero-lateral surface of the arytenoid cartilage. The vestibular folds are frequently used by dogs during an aggressive body posture.

The sound " $\mathrm{b}$ " can be emitted alone, or before the "é*" vowel, and it always has the transitional attack and the duration of emission is also very short. It is emitted when the dog is calling to a child or another animal of the group, his friend, or the owner. The frequency emitted is medium-high, but above all, the intensity is very low, since the sound is directed to be heard in a nearby area. From the behaviour of the dog it could mean: "Where are you?" So the "phonos" $b^{\star}$ and $b^{*}-e^{\star}$ become phonemes and acquire semantic values.

The interrogative sequences, with a low emission are the following:

$$
1] \rightarrow\left[b^{*} \text {, pause, pause, } b^{*} \text {, pause, pause }\right]
$$

or, if the animal being called, or the owner, is inside the territory of the dog, but farther away, we can have the following sequence:

$$
2] \rightarrow\left[b^{\star} e^{\star}, b^{\star} e^{*}, \text { pause, } b^{\star} e^{\star}, b^{\star} e^{\star}, \text { pause }\right]
$$

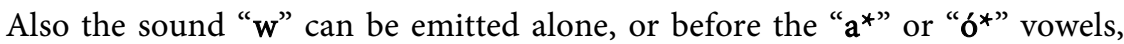
and it always has the transitional attack and the duration of emission is also very short. It can be emitted with a higher frequency than with $\mathbf{b}^{*}$, so it is suitable for vocalizations addressed to more distant individuals belonging to the same group and not to enemies, identified with the sense of smell and not with sight.

So the "phonos" " $w$ " and $w^{*}-a^{*}$ become phonemes and acquire semantic values. So, the sequence is:

$3] \rightarrow\left[\mathrm{w}^{*}\right.$, pause, pause, $\mathrm{w}^{*}$, pause, pause $]$, or

$4] \rightarrow\left[\mathrm{w}^{\star} \mathrm{o}^{\star}\right.$, pause, pause, $\mathrm{w}^{\star} \mathrm{o}^{\star}$, pause, pause $]$

\begin{tabular}{|c|c|c|c|}
\hline \multicolumn{2}{|c|}{ Short vowels } & \multicolumn{2}{|c|}{ Long vowels } \\
\hline$a^{*}$ & $\begin{array}{l}\text { Almost similar to the Italian } \\
\text { word albo }\end{array}$ & & \\
\hline $\mathrm{e}^{*}$ & $\begin{array}{l}\text { Almost similar to the first } \\
\text { vowel "e" not accented of the } \\
\text { French word fenêtre }\end{array}$ & $\mathrm{u}^{*}$ & $\begin{array}{l}\text { Almost similar to the } \\
\text { English word group }\end{array}$ \\
\hline $\mathrm{ó}^{*}$ & $\begin{array}{l}\text { It is it is always followed by } \\
\text { the vowel } U\end{array}$ & í:* & $\begin{array}{l}\text { Almost similar to the } \\
\text { English word sea }\end{array}$ \\
\hline
\end{tabular}

Table 9. Vowels emitted by dogs. 
Table 10. Diphthongs (double vowel sounds) emitted by dogs.

\begin{tabular}{cc}
\hline & Ascendant diphthongs \\
\hline $\mathrm{ua}^{*}$ & Almost similar to the Italian words guàdo, quàdro \\
uo $^{*}$ & Almost similar to the Italian words uòmo, uòvo, buòno \\
\hline
\end{tabular}

Table 11. Consonants emitted by dogs.

\begin{tabular}{l} 
Bilabial occlusive consonant \\
\hline $\mathrm{b}^{*} \quad$ Almost similar to the English word job \\
Velar occlusive consonant \\
Nasal consonant \\
Vibrant alveolar consonants \\
Almost similar to the English word one \\
$\mathrm{w}^{*} \quad \begin{array}{l}\text { The } \mathrm{n}^{*} \text { is emitted with a high position of larynx and it is almost similar to } \mathrm{n} \\
\text { pronounced as in the English word thanks. } \\
\text { When } \mathrm{n}^{*} \text { is immediately followed by the sound } \mathrm{r}^{*} \\
\text { we can have a form of growling, with a sonorous occlusive glottal position. }\end{array}$ \\
$\mathrm{n}^{*} \quad$
\end{tabular}

If the animal is perceived at an olfactory level and also seen, but is not part of the group, the sequence is as follows:

$5] \rightarrow[\mathrm{w} * \mathrm{a} *$, pause, pause, $\mathrm{w} * \mathrm{a} *$, pause, pause, $\mathrm{w} * \mathrm{a} *$, pause, pause $]$

The barking is more excited. The semantic value is "Who are you? What are you doing in my area?".

If the intruder does not go away, then the sequence becomes more articulate and aggressive, as follows:

$$
6] \rightarrow\left[w^{*} o^{*} \cup \overline{a^{*}}, w^{*} o^{*} \cup \overline{a^{*}}, w^{*} o^{*} \cup \bar{a}^{*}\right]
$$

In all these sequences, there is a rhythm, a ternary rhythm, with pulsation over time of that communication, identical for each dog, in the sense that a dog can start shortly after barking and maintain a constant ternary rhythm, with speed compared to that of the other different dog, depending both on the proximity to the intruder, or on the excitement given by the character of the dog and the breed. It follows an overlap of sounds that has a very precise "musical" order, in polyrhythm.

Considering the sequence 5 , we observe that the sound is emitted on the strong time, that is on the time that has the strong accent, followed by two pause times, we could take it as a $3 / 8$, with the speed more or less agitated depending on the situation.

The sequence 6 presents the first sound very fast, then made on an unaccented time, this sound becomes a "glissato" with an accented open sound that is higher. For every each emission $\mathbf{w}^{\star} \mathrm{o}^{\star} \cup \mathbf{a}^{*}$, there is an inhalation. The ratio between 
the frequency of the second sound and that of the first is of a musical interval of an ascending interval of the perfect fourth. It is interesting to observe that the rhythm is as an iambic metric.

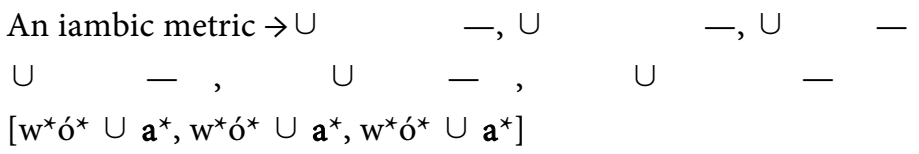

It is formed by an "arsi", a short unaccented syllable and a "thesis" of a long syllable, thus formed of two elements, whereas the iambic metric is formed of three elements (in Latin language "morae"). This is a ternary rhythm, but the iambic metric is appropriate to a situation of excitement, of warning of aggression, even of fighting games, but in this case the posture clearly indicates the game; sequence 6 is also issued by hunting dogs, when they have identified the prey.

If an intruder arrives downwind, so the dog cannot perceive it with its nose, but suddenly sees it and is surprised, a sequence that can often be heard is the following:

$$
\text { 7) } \rightarrow\left[w^{\star} \bar{u}^{\star} \cup w^{\star} u^{*}, w^{*} u^{\star} \bar{U} w^{*} u^{*}, w^{*} u^{\star} \cup \bar{w}{ }^{*} u^{\star} w^{\star} \bar{u}:{ }^{*}\right]
$$

The graphic signal $\cup$ shows a "glissato" between two sounds of these emitted by dogs.

We still have a tripartite rhythm sequence. The sounds are all pitched on the same medium-high frequency, while the last sound sometimes falls with a descending interval of a major third. The semantic translation could be "Go away!".

The sound $\mathrm{k}^{*}$ is emitted with the rapid alternation of opening and closing of the jaws, as an intimidating expression during the competition to maintain the role of alpha dog or wolf. The subject is strongly stressed and surrounded, as old or ill.

When $\mathrm{k}^{*}$ is followed by a sort of $\mathrm{i}^{*}$ thanks to the closure of the jaws and the simultaneous widening of the corners of the mouth and displacement against the hard palate of the tongue, the cry of real pain or simulation is created.

The consonant $\mathbf{m}^{*}$ is emitted in a relaxing situation as a whimpering or with an attempt to imitate a musical sound (this last observation is related to dogs $\mathrm{n}$. $2,5,6,21$, whose owners are musicians).

From the forms of verbal communications of dogs and from their body postures, we can deduce that in the use of this or that other vowel or consonant, the distance of the subject with whom the dog is communicating is already implicit. Moreover, numerous basic semantic vocal functions of verbal communication are obtained, as shown in the following list:

1) query function on where a member of the group is,

2) query function on who is a member who does not belong to the group,

3) query function on what a non-animal sound is,

4) communication function on the position in its territory,

5) communication function on one's own hierarchy (of the alpha dog),

6) function of peremptory communication of intimidation if one violates one's own territory, 
7) peremptory function command communication to go away,

8) function of communication to your group about a situation, for example: the postman arrives, or a fox arrives, I have blocked the prey, the ground is "growling" (in case the subsoil emits infra-sounds or seismic waves arrive),

9) joyful communication of the arrival of a group member (master, human family member, partner...),

10) sad communication of departure of the owner or a member of the group.

Before considering in depth the vocal sequences that are interesting for the connection with the anomalies of some geophysical parameters of the subsoil, it is good to understand why in the vocal language of dogs there are sequences with characteristics of tonal language, with precise frequencies and therefore with relationships between the sounds that determine a semantic nature in the concatenations of the same.

The emissions of the sounds can be detached or tied, with a rhythmic pattern that repeats itself by analogies of situations, regardless of the races. The breed and therefore the size of the dog intervenes only in the height of the sounds (higher for the small breeds), not in the frequency ratios between the intervals.

We have seen how the anatomy and physiology of the dog's phonation limits the articulation of the sounds, its tongue and facial muscles do not allow a language rich in sounds and phonemes differing from one another, so nature selects ways of diversification of language based on rhythmic patterns and frequency. Moreover, the very long collaboration of dogs with mankind implies a language necessarily articulated also according to the needs of the master, as well as for the group life of the dog: the dominance of the master's needs over those of dog life makes the difference between the richer development of dog barking compared to that of the wolf's howling, which therefore only follows the necessities of group life independent of mankind.

There is still to consider that the need to create sounds that are not immediately dispersed in the open environment of the countryside and pastures, in time selects a language that is intoned and less spoken. Also the semantic content of a sentence is better identified in the distance if it has rhythmic patterns. Just think of the particular language that the shepherds of sheep and of cows have with their dogs and their grazing beasts: they are interjections like "ouuh", "eiih", accompanied by signs of the sticks that indicate the direction to be taken. I have observed this language for a long time, in the midst of their dogs and flocks and herds. It is amazing to observe that such human sounds are emitted with an unconscious ternary rhythmic pattern. This is a winning scheme regarding distance, for men and animals.

\section{The Earth's Language}

\subsection{Physical and Chemical Parameters and Anomaly Detection}

The study of the structure of the ionosphere, the lithosphere, the atmosphere, the dynamics of fluids, the internal structure of the globe, that of earthquakes 
and volcanoes, geothermal, geomagnetic field, solar radiation, and so on, leads inevitably to the observation on how variations of some physical and chemical parameters in a certain area of the globe can interact with the complex synergistic system of the Earth.

Continuous monitoring of physical and chemical parameters can therefore give information on the performance of that parameter over time, offering the possibility of identifying any anomalies in short, medium or long periods, with mathematical criteria.

Then, the study of possible seismic precursors implies a multi-parametric and possibly continuous monitoring in order to evaluate "in retrospect" the possible occurrences of anomalies preceding seismic events. Ideally it would be possible to create multi-parameter monitoring Centers that can share data and for some parameters, such as variations of magnetic declination, to be able to make a triangulation. The literature is rich in observations of pre-seismic anomalies, such as variations on water reservoirs, pre-seismic and seismic lights, electrical and magnetic anomalies, even in swarms, significant emissions of radon, and unusual behaviour of animals. However, the non-continuity of the observations makes it very difficult if not impossible to attribute an anomaly to a given moment in the earthquake nucleation process.

If an anomaly precedes the seismic event by many hours or days, it can go unnoticed. For example, for the warnings of dogs, we remember those that immediately precede the earthquake (about 40 seconds before), that is the perception of the seismic waves " $p$ ", but then they are not precursors. Those of a few hours before are not remembered, because they are confused with the normal barking of dogs. So after an earthquake important for its magnitude, the questionnaires proposing that people improve their knowledge on the precursors do not contain precise observations made by the people on the anomalies of the animals, observed a few hours before.

To evaluate any correlations between the anomalies of some monitored parameters, we proceeded to the chronological recording of a few parameters $y(t)$, with monitoring that was not always uniform, in order to extend a background for each of them, on which to then apply statistical evaluation techniques.

To consider the behaviour of a variable $y(t)$ observed experimentally during the continuous monitoring of a historical series $y(t)$ anomalous, I considered the mean values in day, night, monthly, and seasonal time intervals, also considering the parameters of the weather, so as to be able to identify the "normal" behaviours of the considered parameter. I then made groupings, classifications, to be able to identify punctual, contextual, and possibly collective anomalies.

There are some parameters to be measured for the identification in the vocalizations of dogs: the frequencies of the sounds, the relationships between the intervals of the emitted sounds, the rhythmic sequences referred to a constant pulsation of time, allowing to parameterise the vocalization, with the consequent identification of anomalies in dog communications, referring to the contexts of other measured parameters. 
The moderate seismicity of the zone allows to identify, with long periods of monitoring some parameters, the behaviour of the same in temporal intervals without earthquakes and in those with earthquakes. The same anomaly of a measured variable, which occurs experimentally several times within a certain time before an event, leads me to consider it to be a probable precursor of that event in the medium or short term.

Western Piedmont is an area with a variable magnetic variation from point to point, similar to the area surrounding the volcano Etna and Catania (in Sicily, Italy) and to that in front of the volcano Vesuvius, near Naples. This is visible in the geographical map of the image 4 .

This feature makes the comparison between the geomagnetic anomalies recorded in Val Pellice and the vocal sequences of dogs contemporaneous to them, even more interesting. In fact, in the presence of such anomalies, dogs bark with specific sound frequencies and with distinguishable rhythmic characterization.

In a multi-parametric monitoring it is useful to know the instrumental typology and the data collection modality, as shown in Table 12 and in Table 13, in order to compare the possible physical anomalies with the unusual behaviors of the animals, in this case of the dogs. Mainly fundamental for the semantic detection of some vocal sequences is the possibility of temporally associating them with the appearance of physical anomalies, circumstances observed a very high number of times in order to rise to the semantic value.

Figure 4 shows the map of the magnetic declination anomalies recorded by the INGV of Rome, but the map is a reworking of the Author, taken from the INGV declination magnetic card (2015), revision to better highlight the isogonic lines and the area where the magnetic declination varies from point to point.

Looking at Figure 4 we can see how the SPSC Studies Center, in Torre Pellice, is included in the yellow area, where the magnetic declination changes from point to point. Similar areas are located near Vesuvius, Etna and in some other areas, including Sardinia. We can also note the isogonic lines with values of 6.5 , 7.0, 7.5, 8.0 arc minutes and three of the Italian INGV Observatories.

The colours, shown on the right of the map, correspond to the magnetic declination values, measured in arc minutes, placed to the right of the coloured column.

The INGV Observatories for analysis and collection of magnetic data are the following, in Italy:

- L'Aquila Observatory, IAGA Code AQU,

- Castello Tesino Observatory, IAGA Code CTS,

- Lampedusa Observatory, IAGA Code LMP,

- Duronia Observatory, IAGA Code, DUR.

Only three of the four Italian Observatories are visible on the map.

In Australia there are two of the other Observatories, with the related IAGA codes:

- Mario Zucchelli Observatory, IAGA Code TNB,

- Concordia Observatory, IAGA Code DMC. 
Table 12. Physical parameters monitored in SPSC.

\begin{tabular}{|c|c|c|}
\hline Physical parameter & Instrumentation & Measurement unit and sensitivity \\
\hline Magnetic induction & 1 TreField EM Meter & $\begin{array}{c}0.1-100 \mu \mathrm{T} \text { in } \\
\text { logarithmic scale }\end{array}$ \\
\hline Magnetic declination $\delta$ & 4 compasses Virginia & $\begin{array}{l}6036 \text { VA Sensitivity of } \pm 0.5^{\circ} \\
\text { minutes of arc }\end{array}$ \\
\hline$\beta, \gamma$ particles & 1 Geiger Ю нчмер SКM 05 & $\begin{array}{l}\text { Scale } 0.1-99.9 \mu \mathrm{S} / \mathrm{h} ; \\
\text { alarm at } 0.5 \mu \mathrm{S} / \mathrm{h}\end{array}$ \\
\hline Radon $222 \alpha$ particles & Geoex, model 1027 & $\mathrm{pC} / \mathrm{l}$ \\
\hline Temperature & $\begin{array}{c}1 \text { analogical thermometer } \\
\text { Degrees Celsius }\end{array}$ & $\left( \pm 0.1^{\circ} \mathrm{C}\right)$ \\
\hline Temperature & 1 thermometer TM-917 DICOM & from $-100^{\circ} \mathrm{C}$ to $+132^{\circ} \mathrm{C}\left( \pm 0.1^{\circ} \mathrm{C}\right)$, \\
\hline $\begin{array}{c}\text { Temperature, } \\
\text { pressure/humidity }\end{array}$ & PCE-FWS 20 & Celsius degrees, $\mathrm{hPa}, \%$ \\
\hline Water $\mathrm{pH}$ & Litmus papers & // \\
\hline Infra-sounds & Infrasonic 200, Aetech & $\mathrm{Hz}$ \\
\hline EM signals & $\begin{array}{l}\text { ELF, VLF and LF CIEN } \\
\text { electrodes }\end{array}$ & $4 \mathrm{~Hz}-50 \mathrm{kHz}$ \\
\hline
\end{tabular}

Table 13. Monitoring modality.

\begin{tabular}{|c|c|c|}
\hline Physical parameter & Monitoring modality & Period of monitoring \\
\hline Magnetic induction & 2 time/day for $1 / 2 \mathrm{~h}$ every time & $1999-2018$ \\
\hline Magnetic declination $\delta$ & $\begin{array}{l}\text { - } 2 \text { time/day for } 1 / 2 \mathrm{~h} \text { every time } \\
\text { - Continuous monitoring for half an hour at } \\
\text { the time of the vocal sequences } 8 \text { and } 9\end{array}$ & $\begin{array}{l}1998-2013 \\
2014-2018\end{array}$ \\
\hline$\beta, \gamma$ particles & $\begin{array}{l}\text { data every second } 2 \text { time/day for } 1 / 2 \mathrm{~h} \\
\text { every time }\end{array}$ & $2003-2015$ \\
\hline Radon $222 \alpha$ particles & $\begin{array}{l}\text { Continuous: PC connection basement, at } 0.30 \\
\mathrm{~m} \text { from the floor }\end{array}$ & 2011-2018 \\
\hline Temperature & $\begin{array}{l}2 \text { time/day at the same hours In cellar and in } \\
\text { Biglione creek }\end{array}$ & $1999-2018$ \\
\hline Temperature & every $0.4 \mathrm{sec}$. Continuous: PC connection & 2013 \\
\hline $\begin{array}{l}\text { Temperature, } \\
\text { pressure/humidity }\end{array}$ & 2 time/day at the same hours & 2013 \\
\hline Water $\mathrm{pH}$ & 1 time/week SPSC/garden/Biglione & $2012-2013$ \\
\hline Infra-sounds & 5 samples/second Continuous: PC connection & 2013-2016 \\
\hline EM signals & Continuous: PC connection & $2012-2013$ \\
\hline
\end{tabular}

The IAGA code is attributed by the International Association of Geomagnetism and Aeronomy, an international scientific organization, established in Rome in 1954.

\subsection{The Variations of the Magnetic Field and Other Anomalies}

During the nucleation phase of earthquakes, the magnetic field variations induce a magnetization orientation on magnetic domains of a ferromagnetic substance. The structure of the crystals influences the direction of magnetization of a 


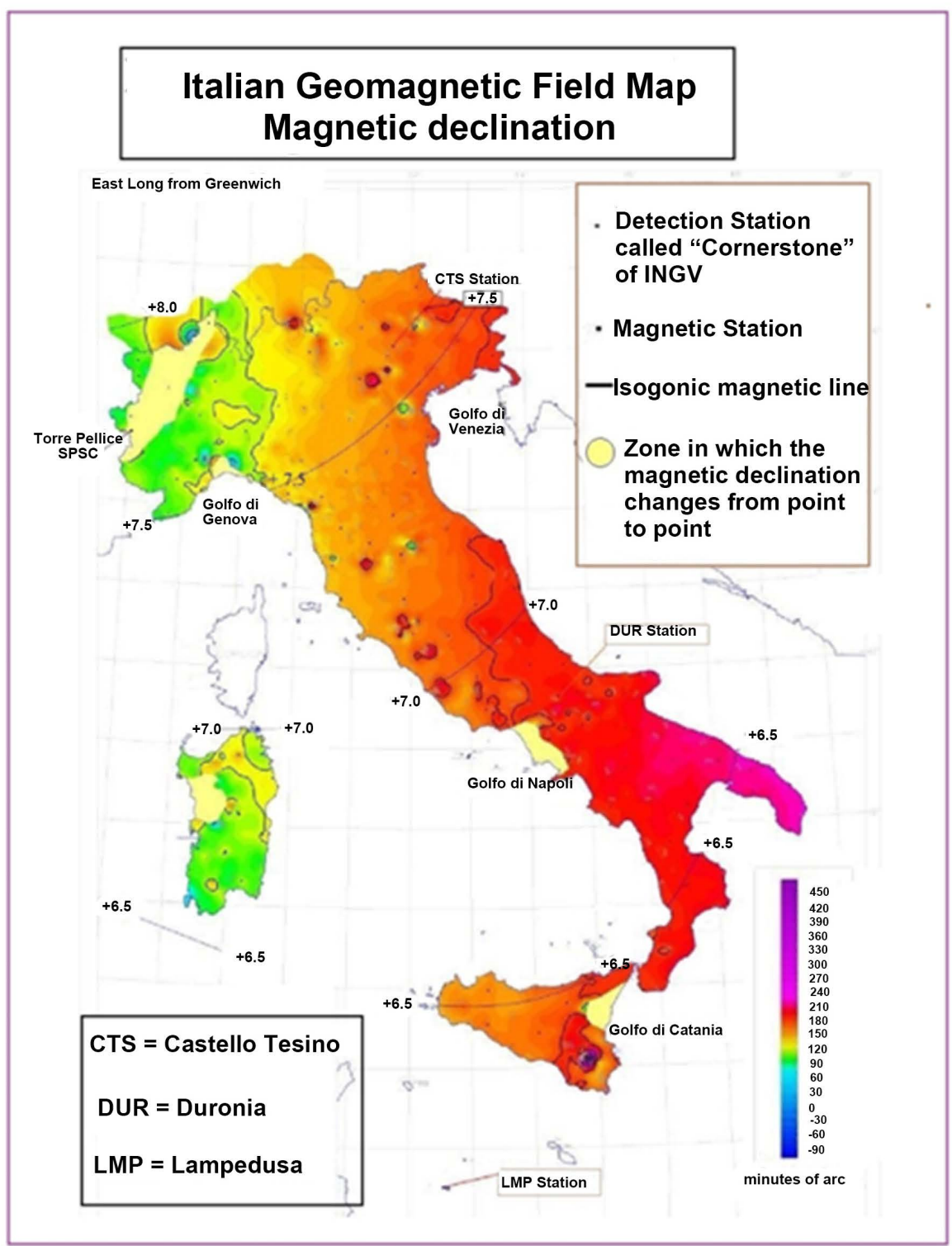

Figure 4. De Liso's elaboration from Italian magnetic declination by "INGV Roma, Cartografia 2015".

domain. The geologic morphology of the Pellice Valley is rich in quartzite and gneiss [28], so in ferromagnetic rocks with iron crystals with a cubic structure (gneiss), the most immediate direction of magnetization is along the three axes of the cube. The magnetic variation of the domains tends to align in the direction of the field as the strength of the external magnetic field increases. If there are also piezoelectric crystals, (quartzite) in the rocks, a distribution of charges is created on crystal surfaces due to the elastic deformation, that can be acoustic, mechanical, and magnetic [29] [30] [31] [32] [33]. During the seismic nucleation, the deep rocks undergo a pressure that can change the intensity of the magnetic permeability $\mathrm{B}$ and the direction of magnetization of the magnetic domains can justify the sudden change I observe of the $\delta$ magnetic declination that I measure in minutes of arc. This precise moment is the sudden magnetic 
commencement and I have to separate it from periods of magnetic variations from solar storms. Magnetic intensity and declination angle values present a gradual peak reduction [34].

So far, for each earthquake considered, with distance from the epicentrum of earthquakes from the SPSC less than $100 \mathrm{~km}$ and with magnitude even lower than $\mathrm{ML}=1$, I noticed that, from the moment of sudden commencement to the earthquake the variations in intensity of the geomagnetic field, magnetic declination, and temperature on the ground, measured half a meter beneath the floor of the SPSC cellar, until their almost zero decrease, there is the same interval of time, which is longer if the future earthquake is farther away. Obviously this is known "in retrospect", with the comparison of the Italian seismic INGV list. Radon also has particular intervals of increase and decrease before earthquakes, but they do not always coincide with those of magnetism and temperature [35]-[40]. The comparative study of multiple anomalies of the physical parameters considered has led me to identify three pre-seismic moments, or phases, according to the following scheme, in Table 14, each of which presents recurring characteristics for the unusual behaviours of dogs.

Some clarifications will better explain the contents of Table 14: as regards box 1 of phase $B$, my instrument records values in $\mu \mathrm{T}$ and not in $\mathrm{nT}$; for every earthquake studied, I have always observed the anomalies described in the boxes $1,2,3,4,5$, and 13 of phases $A_{1}, A_{2}$ and $B$.

So we can say that at least these anomalies can be considered seismic precursors, with a certain degree of forecast (not predictability) in the long or medium or short term. The anomalies described in phase $A_{1}$ may occur a few days, or weeks, before the first seismic shock and therefore present themselves as long-term temporal precursors. Only continuous multi-parametric monitoring can help to distinguish the various phases.

Having identified physical parameters concerning their vocal communication has made their barking semantic, thereby the observation of dogs is precious and fundamental, and very useful for the study of seismic precursors.

\section{Some Seismic Precursors and Vocal Reactions of Dogs}

If a lengthy analysis of the multi-parametric monitoring in a moderately seismic zone, together with the observation of other anomalies of the territory and of the unusual behaviour of the animals, has led to observe precise recurrences of anomalies before seismic events, it can be said that those anomalies belong to a phase of preparation of local earthquakes and therefore can be identified as seismic precursors, also thanks to the fact that the moderate seismicity allows to separate seismic nucleation intervals from others without earthquakes.

The difficulty consists in discerning the nucleation of future earthquakes with great magnitude from other rooms, of small magnitude seismic events that often precede these disastrous events by a few hours. This happened for the earthquakes in Emilia in 2012. 
Table 14. Three pre-seismic phases from sudden magnetic commencement to the first shock of earthquakes.

\begin{tabular}{|c|c|c|}
\hline Phase $A_{1}$ & Phase $\mathrm{A}_{2}$ & Phase B \\
\hline $\begin{array}{l}\text { 1) first sudden magnetic } \\
\text { commencement for intensity } \\
\text { variation }\end{array}$ & $\begin{array}{l}\text { 1) almost regular time spans between } \\
\text { peaks of often equal intensity and ever } \\
\text { lower peaks }\end{array}$ & $\begin{array}{l}\text { 1) there are no } \\
\text { variations observed } \\
\text { in } \mu \mathrm{T}\end{array}$ \\
\hline $\begin{array}{l}\text { 2) first sudden magnetic } \\
\text { commencement declination }\end{array}$ & $\begin{array}{l}\text { 2) almost regular time spans between } \\
\text { peaks of often equal declination angle } \\
\text { and ever lower peaks }\end{array}$ & 2) no variations \\
\hline $\begin{array}{l}\text { 3) variable periods of decreasing } \\
\text { peaks for magnetic values }\end{array}$ & 3) short periods & $/ /$ \\
\hline $\begin{array}{l}\text { 4) the first intensity variation of the } \\
\text { sudden magnetic commencement is } \\
\text { proportional to next local seism's } \\
\text { magnitude ML }\end{array}$ & /I & $/ /$ \\
\hline $\begin{array}{l}\text { 5) variations in temperature in the } \\
\text { subsoil }\end{array}$ & 5) decrease & $\begin{array}{l}\text { 5) no variations above } \\
\text { the seasonal average }\end{array}$ \\
\hline 6) there may be a powder emission & 6) decrease in rapport to $\mathrm{A} 1$ & 6) more decrease \\
\hline 7) there may be Radon ${ }^{222}$ emission & $\begin{array}{l}\text { 7) there may be high Radon } \\
\text { emission and then a rapid decrease }\end{array}$ & $\begin{array}{l}\text { 7) no variations above } \\
\text { the weekly average }\end{array}$ \\
\hline $\begin{array}{l}\text { 8) there may be a water } \mathrm{pH} \\
\text { variation }\end{array}$ & 8) decrease in rapport to $A_{1}$ & $\begin{array}{l}\text { 8) more decrease in } \\
\text { rapport to } A_{1}\end{array}$ \\
\hline $\begin{array}{l}\text { 9) there may be variations of the } \\
\text { water reservoirs }\end{array}$ & $\begin{array}{l}\text { 9) there may be variations of the water } \\
\text { reservoirs }\end{array}$ & $\begin{array}{l}\text { 9) there may be } \\
\text { variations of the water } \\
\text { reservoirs }\end{array}$ \\
\hline $\begin{array}{l}\text { 10) there may be emissions of some } \\
\text { gases in the air or in the reservoirs } \\
\text { of water }\end{array}$ & $\begin{array}{l}\text { 10) there may be emissions of some } \\
\text { gases in the air or in the reservoirs of } \\
\text { water }\end{array}$ & $\begin{array}{l}\text { 10) decrease in rapport } \\
\text { to } \mathrm{A} 1\end{array}$ \\
\hline $\begin{array}{l}\text { 11) there may be weather } \\
\text { variations, with luminous } \\
\text { phenomena }\end{array}$ & $\begin{array}{l}\text { 11) there may be weather variations, } \\
\text { with luminous phenomena }\end{array}$ & $\begin{array}{l}\text { 11) there may be } \\
\text { weather variations, } \\
\text { with luminous } \\
\text { phenomena }\end{array}$ \\
\hline $\begin{array}{l}\text { 12) electrical and electromagnetic } \\
\text { variations }\end{array}$ & 12) decrease & 12) more decrease \\
\hline $\begin{array}{l}\text { 13) unusual behaviour of dogs } \\
\text { concerning both non-verbal } \\
\text { communication and vocal } \\
\text { sequences }\end{array}$ & $\begin{array}{l}\text { 13) unusual behaviour of dogs } \\
\text { concerning both non-verbal } \\
\text { communication and vocal sequences }\end{array}$ & $\begin{array}{l}\text { 13) only vocal } \\
\text { sequences of an alpha } \\
\text { dog of an over-group } \\
\text { of groups }\end{array}$ \\
\hline $\begin{array}{l}\text { 14) there could be some unusual } \\
\text { behaviours of other animals, which } \\
\text { depend on the anomalies } \\
\text { mentioned above }\end{array}$ & $\begin{array}{l}\text { 14) there could be some unusual } \\
\text { behaviours of other animals, which } \\
\text { depend on the anomalies mentioned } \\
\text { above }\end{array}$ & $\begin{array}{l}\text { 14) there are no vocal } \\
\text { alarms, eventually } \\
\text { possibly only health } \\
\text { problems for certain } \\
\text { species }\end{array}$ \\
\hline
\end{tabular}

I also observed what the dogs have reported with vocal alarms simultaneously to their productions, the following precursor anomalies:

1) the magnetic anomalies,

2) the electromagnetic ELF variations,

3) phenomena expressed with infra-sounds or with audible sounds such as the "brontide" or Mistpouffers or skyquakes. 


\subsection{The Magnetic Anomalies and Vocal Sequences of Dogs}

Having found that every time there is a magnetic anomaly of declination, even small, the dogs punctually state the marker with a particular language, has led me to think that even dogs, like many other animals, have some element of their body that is magneto-sensitive, not only to the variation of intensity, but above all, that of declination. In 2013, the researchers Petra Novákova, Erich Pascal Malkemper and others, proved the magnetic sensitivity in dogs. The conclusion of their paper states [41]: "magnetic sensitivity was proved in dogs, a measurable, predictable behavioural reaction upon natural MF fluctuations could be unambiguously proven in a mammal, and high sensitivity to small changes in polarity, rather than in intensity, of MF was identified as biologically meaningfur".

Christine Nießner and Leo Peichl from the Max Planck Institute for Brain Research in Frankfurt and other researchers from the Ludwig-Maximilians University in Munich, the Goethe University in Frankfurt, and the Universities of Duisburg-Essen and Göttingen have conducted research on the Cryptochromes 1 in birds and in mammals [42]. These light-sensitive molecules exist in bacteria, plants, and animals and they are involved in the control of the body's circadian rhythms. Cryptochrome 1a is located in photoreceptors in birds' eyes and is activated by the magnetic field.

The research found Cryptochrome 1 only in a few species from the carnivore and primate groups. The active Cryptochrome 1 is found in the light-sensitive outer segments of the cone cells.

As is the case in birds, it is found in the blue-sensitive cones in dogs, wolves, bears, foxes, and badgers, but it is not found in cat-like carnivores such as cats, lions and tigers.

This scientific research gives me an anatomical-physiological explanation for the reactions of dogs to changes in the magnetic field (MF) that I have observed for years before the earthquakes.

As we can see in Table 13, the magnetic anomalies of both declination and intensity of the FM occur suddenly and frighten the dogs, who do not see the "enemy" as when they see an intruder in their territory, but perceive the magnetic change with their magneto-sensitive receptors and are disoriented, just as the birds are also disorientated, and which in these moments can "make mistakes" in the direction of flight and go slamming into plants. Dogs then raise their snouts to sniff better, as if this event even brings them their own smells.

Sometimes they get up on their hind legs and while spinning around, they look up and bark, but they do not know in what direction they have to bark to warn off the "enemy". Stress involves a considerable energy of breath emission, so vocalizations are "pushed", still using the musical jargon and so the interrogative sequences, like the sequence n. 6

$$
6] \rightarrow\left[w^{*} \dot{o}^{*} \cup \overline{\mathbf{a}^{*}}, \mathrm{w}^{\star} \mathbf{o}^{*} \cup \overline{\mathbf{a}^{*}}, \mathrm{w}^{\star} \mathbf{o}^{*} \cup \overline{\mathbf{a}^{*}}\right]
$$

no longer imply intervals of fourth just between the first and the second sound, 
but intervals of fourth excess $(\Lambda \uparrow *)$, first, during phase $A_{1}$, with binary rhythms issued several times (sequence 8 ),

$$
8] \rightarrow\left[\mathrm{w}^{\star} \mathrm{o}^{\star} \cup \mathrm{a} \uparrow{ }^{*} \text {, pause, } \mathrm{w}^{\star} \mathrm{o}^{\star} \cup \mathrm{a} \uparrow{ }^{*}\right. \text {, pause,... }
$$

and so on

then, as aggression increases, during phase $A_{2}$, following ternary rhythm and with an iambic accent of the sequence n. 9:

$$
9] \rightarrow\left[w^{*} o^{*} \cup \bar{a} \uparrow{ }^{*}, w^{*} o^{*} \cup \bar{a} \uparrow{ }^{*}, w^{*} o^{*} \cup \bar{a} \uparrow{ }^{*}\right]
$$

Preceded by the alpha dog of the super group observed, (see below) all the dogs bark together, each with their own rhythm, based on the intensity of agitation, and along with the dogs, the roosters and hens, blackbirds, magpies, and crows are also complaining.

As the compass needle returns to the damped harmonic oscillation of the previous values, the sequences of the type 9 diminish and overlap them with the sequence similar to that aimed at the intruder of the no longer aggressive injunction to leave:

$$
\begin{aligned}
10] \rightarrow & {\left[\mathbf{w}^{*} \mathbf{u}:^{*}, \text { short pause, } \mathbf{w}^{*} \mathbf{u} *^{*},\right. \text { short pause, }} \\
& 1^{\text {th }} \text { sound } 2^{\text {nd }} \text { sound } \\
& \mathbf{w}^{*} \mathbf{u}:^{*} \text {, short pause, short pause, short pause, } \\
& 3^{\text {rd }} \text { sound } \\
& \mathrm{w}^{*} \mathrm{u}:{ }^{*}, \quad \mathrm{w}^{*} \mathrm{u}:{ }^{*}, \text { short pause } \mathrm{w}^{*} \mathrm{u}: \\
& 4^{\text {th }} \text { sound, } \quad 5^{\text {th }} \text { sound, } \quad 6^{\text {th }} \text { sound }
\end{aligned}
$$

if we call $v_{1}$ the frequency of the fundamental of the first sound, $v_{2}$ of the second sound, and so on, the ratio between the frequencies are as follows:

$$
\begin{array}{ll}
\text { c] } \rightarrow & v_{1} / v_{2}=9 / 8 \\
\text { d] } \rightarrow & v_{2} / v_{3}=10 / 9 \\
\text { e] } \rightarrow & v_{4} / v_{1}=10 / 9 \\
\text { f] } \rightarrow & v_{5}=v_{1} \\
\text { g] } \rightarrow & v_{6} / v_{5}=5 / 4
\end{array}
$$

The sequence 10, with the first three sounds in descending scale, shows the sound ratio of a Pitagora's scale. The alpha dog of the super group (see below) that I have studied always begins this sequence at the end of phase $A_{2}$, always from the note $G_{3}$, central in the piano, this intonation of sounds is astonishing. So the notes are: $G_{3}, F_{3}, E_{3}(b), A_{3}(b), G_{3}, B_{3}$.

On the basis of experience, I have observed that the shorter the epicentrum distance of an earthquake occurred from the SPSC center, the shorter the time intervals of phases $A_{1}, A_{2}, B$, especially of phase $B$, have been (with retrospective control) and even more numerous was the list of anomalies of the various parameters described in the boxes $A_{1}$ and $A_{2}$. The longer the distance of the epicentrum of an earthquake occurred from the SPSC Center, the longer the time interval of phase $A_{1}$.

If at sudden commencement the intensity values of the geomagnetic field are high (for example $0.40-50 \mu \mathrm{T}$ ) and the maximum amplitude angle of the declination variation is achieved over a time interval greater than 100 minutes, it is 
probable that a medium-high magnitude earthquake is being prepared with the area of nucleation at a distance of more than $200 \mathrm{~km}$ from the observation Center. If, on the other hand, together with high geomagnetic intensity values, the maximum amplitude angle of the declination variation is achieved over a time interval of less than 10 minutes, generally a medium-high magnitude earthquake could happen within approximately $50 \mathrm{~km}$.

Now, in Table 15, I'll give some examples regarding each of the earthquakes for which the dogs have sent vocal alarms both for the phase $A_{1}$ and $A_{2}$, while for the phase $\mathrm{B}$, only the alpha dog of the super group has complained before each earthquake.

In an area that little populated, with scattered peasant houses with farm animals, there are also dogs of different races, often crossbreeds, and many guard dogs trained to manage the flocks: these, free to roam over a vast territory, create a super group, in defence of its territory and its "own" against wild boar, foxes, and wolves. This group therefore includes as subgroups the furthest areas of those dogs living in the villas, enclosed in the yard, without being able to go out except when they run away. The occasional escape creates the opportunity to relate to free dogs. Then latter often go to find the dogs after they've returned to being enclosed in the yards. The continuity of the relationship is subsequently based on vocal communication, which becomes more important than the binomial posture-vocal communication. So, the alpha dog of the super group also becomes the alpha dog of the sub-groups. Therefore, it has the right to raise the alarm for any enemies that may endanger all members of the super group, at simultaneous times and not as usually happens for intruders who upon their arrival to this or that area are reported by dogs in operation of the path that the intruder takes. The magnetic anomalies before the earthquakes are parameters that should be studied by several Centers of multi-parametric monitoring, in order to develop a deeper knowledge on the nucleation processes of earthquakes.

Table 15. Vocal alarms both for the phase $A_{1}$ and $A_{2}$.

\begin{tabular}{|c|c|c|c|c|c|c|}
\hline Local Time & $\begin{array}{l}\text { Event } \\
\text { location }\end{array}$ & $\begin{array}{c}\text { ML } \\
\text { Depth km }\end{array}$ & $\begin{array}{l}\text { mag. Initial intensity } \\
\text { variation }\end{array}$ & $\begin{array}{l}\text { Initial } \delta \text { time } \\
\text { Phase } A_{1}\end{array}$ & $\begin{array}{l}\text { Distance to } \\
\text { SPSC Km }\end{array}$ & Initial $\delta$ of $A_{1}$ \\
\hline $\begin{array}{c}2014-04-07 \\
21: 26: 59\end{array}$ & Saint-Paul-sur-Ubaye & $\begin{array}{l}\mathrm{ML}=4.9 \\
8 \mathrm{~km}\end{array}$ & $0.35 \mu \mathrm{T}$ & $\begin{array}{c}\text { h. 10:13' } \\
673^{\prime} 59^{\prime \prime} \\
\text { before seism }\end{array}$ & 50.11 & $5 \mathrm{E}$ \\
\hline $\begin{array}{c}2015-09-20 \\
09: 32: 08\end{array}$ & Villar Pellice & $\begin{array}{c}\mathrm{ML}=3.1 \\
12 \mathrm{~km}\end{array}$ & $0.20 \mu \mathrm{T}$ & $\begin{array}{c}\text { h. } 02: 19 \\
433^{\prime} 08^{\prime \prime} \\
\text { before seism }\end{array}$ & 2.98 & $0.5 \mathrm{~W}$ \\
\hline $\begin{array}{l}\text { 2015-11-06 } \\
\text { 05:03:04 }\end{array}$ & La Condamine-Chatelard & $\begin{array}{c}\mathrm{ML}=3.8 \\
11 \mathrm{~km}\end{array}$ & $0.30 \mu \mathrm{T}$ & $\begin{array}{c}\text { h. } 17.35 \\
868^{\prime} 4^{\prime \prime} \\
\text { before seism }\end{array}$ & 51.25 & $6 \mathrm{E}$ \\
\hline $\begin{array}{l}2016-07-30 \\
22: 21: 38\end{array}$ & Inverso Pinasca & $\begin{array}{c}\mathrm{ML}=3.9 \\
15 \mathrm{~km}\end{array}$ & $0.30 \mu \mathrm{T}$ & $\begin{array}{c}\text { h. } 12.03 \\
618^{\prime} 38^{\prime \prime} \\
\text { before seism }\end{array}$ & 14.82 & $2 \mathrm{E}$ \\
\hline $\begin{array}{c}2018-03-27 \\
15: 29: 49\end{array}$ & Lusernetta & $\begin{array}{c}\mathrm{ML}=3.0 \\
16 \mathrm{~km}\end{array}$ & $\begin{array}{c}0.20 \\
\mu \mathrm{T}\end{array}$ & $\begin{array}{l}\text { h. } 5.30 \\
629^{\prime} 49^{\prime \prime}\end{array}$ & 3.74 & $1.5 \mathrm{E}$ \\
\hline
\end{tabular}


Table 16 shows the seismic list and then Graphic 1 shows the magnetic declination that occurred in August 2018.

We can observe in Graphic 1 that there is a remarkable ascending magnetic declination peak.

The values found in the morning of August 16 quickly went from $7^{\circ} \mathrm{E}$ to $14^{\circ} \mathrm{E}$, with these variation times: at $8: 25$ the delta angle was at $7^{\circ} \mathrm{E}$, at $8: 26$ it was at $9^{\circ} \mathrm{E}$, at 8:27 it was still at $7^{\circ} \mathrm{E}$, at $8: 45$ it was at $14^{\circ} \mathrm{E}$.

My dogs, visually observed and paying attention to the others to discern the various dogs and their vocalizations acoustically, immediately manifested discomfort with their own barking that they emit at the moment of events that surprise them and that they cannot define, which will be discussed more deeply in the next chapter. At h. 20:07:13, an earthquake of $M L=2.1$ occurred in France, at a distance in a straight line with the Center of $90.02 \mathrm{~km}$. Fortunately, the earthquake that occurred came after the declination peak had a small magnitude, but I think that for dogs the discomfort is caused by the considerable variation of declination and by the variation in intensity of the magnetic field. In this case the values recorded at the sudden commencement were $0.20 \mu \mathrm{T}$ and then they gradually fell.

Another example of the alarm of the dogs and other species as well, is given by the intense anomaly of declination and magnetic intensity registered suddenly on June 5,2009 , at h.10.30'. In the graph number 2 the $A_{1}$ phase is short, followed by a longer $A_{2}$ with regular peaks with the same values. The declination is toward West.

The declination values of Graphic 2 towards the West are considered positive. The variations of declination are to be referred to the position of the compass needle that the local North provides to me.

At the sudden commencement, some female blackbirds bumped into the wall of the SPSC and the dogs emitted multiple vocal alarms, which repeated every time the peaks were ascending and not descendants. The phase B, announced by the alpha dog of the super group, began in the morning of June 8 at 8:38 am, the earthquake occurred at 23:35:32, on the border with France less than $90 \mathrm{~km}$ away.

By June 28, it had been followed by 10 earthquakes, for which Torre Pellice was located in relation to the relative epicentres in the Center of a sunburst towards the south-west, with an area with a radius of less than 90k. The seismic sequence showed increasingly minor declining anomalies, always reported by the dogs. I have yet to observe that when registering zero values both for declination and intensity magnetic variables, there may be variations of degree lower than $0.5^{\circ}$ for declination or intensity values in the $\mathrm{nT}$ scale, while I register in $\mu \mathrm{T}$. The seism occurred on June 28 , at 4:14:47 a.m., with $\mathrm{ML}=2.6$, the earthquake on June 28 had been announced by the alpha dog of the super group 4 hours earlier, at the beginning of phase B. 
Table 16. Seismic events occurred in an area of radius $r$ less than $100 \mathrm{~km}$ from SPSC, August 2018.

\begin{tabular}{|c|c|c|c|c|c|}
\hline Time UTC & Latitude & Longitude & Depth km & ML & Event location \\
\hline 2018-08-02Т03:05:35 & 44.5647 & 6.8312 & 9.7 & 1.2 & Italian-French border \\
\hline 2018-08-02T14:25:29 & 44.4957 & 7.1197 & 9.7 & 0.7 & Stroppo \\
\hline 2018-08-03T01:21:47 & 44.4957 & 7.0668 & 22.3 & 3.0 & Elva \\
\hline 2018-08-04T04:32:39 & 44.4132 & 7.2972 & 0.8 & 0.5 & Pradleves \\
\hline 2018-08-05T02:37:54 & 45.8003 & 7.4238 & 11.1 & 0.2 & Oyace \\
\hline 2018-08-06T03:07:04 & 45.3305 & 7.4085 & 9.5 & 0.9 & Ceres \\
\hline 2018-08-06T05:35:39 & 44.8373 & 7.4218 & 24 & 1.2 & Macello \\
\hline 2018-08-06T17:00:29 & 45.443 & 6.2997 & 10 & 1.0 & France \\
\hline 2018-08-09T05:53:39 & 44.8988 & 6.636 & 9.6 & 0.9 & Italian-French border \\
\hline 2018-08-09T06:02:58 & 44.2797 & |8.0447 & 7.1 & 0.6 & Bagnasco \\
\hline 2018-08-09T12:27:32 & 45.775 & $7.0878 \mid$ & 9.2 & 1.2 & La Salle \\
\hline 2018-08-10T03:29:36 & 44.8948 & 6.6333 & 10.0 & 1.0 & Italian-French border \\
\hline 2018-08-11T23:53:55 & 44.533 & 7.3617 & 4.4 & 0.9 & Valmala \\
\hline 2018-08-12T00:16:01 & 44.5258 & 7.3027 & 10.0 & 1.2 & Valmala \\
\hline 2018-08-12Т16:29:49 & 44.1667 & 7.8872 & 10.5 & 0.7 & Ormea \\
\hline 2018-08-12T22:13:00 & 44.5062 & 7.0998 & 10.3 & 0.9 & Stroppo \\
\hline 2018-08-13Т00:07:23 & 44.3198 & 7.2813 & 11.0 & 1.5 & Demonte \\
\hline 2018-08-13T11:26:35 & 44.1587 & 7.8892 & 10.4 & 1.1 & Ormea \\
\hline 2018-08-14T20:53:50 & 45.0113 & 7.3558 & 21.5 & 1.1 & Cumiana \\
\hline 2018-08-15T19:03:50 & 44.4572 & 7.0807 & 9.5 & 0.7 & Canosio \\
\hline 2018-08-16T20:07:13 & 45.6338 & 6.5718 & 12.5 & 2.1 & France \\
\hline 2018-08-17T06:52:57 & 45.9062 & 7.4098 & 10.5 & 0.9 & Bionaz \\
\hline 2018-08-17T20:45:32 & 45.8303 & 7.038 & 10.0 & 1.1 & Courmayeur \\
\hline 2018-08-18T12:22:58 & 44.2017 & 7.3033 & 11.0 & 1.4 & Entracque \\
\hline 2018-08-19T14:53:56 & 44.4078 & 7.2347 & 11.9 & 1.5 & Castelmagno \\
\hline 2018-08-20T04:37:31 & 44.8243 & 6.7992 & 13.4 & 1.0 & Italian-French border \\
\hline 2018-08-20T09:05:37 & 44.9047 & 5.7785 & 6.2 & 1.7 & Franch \\
\hline 2018-08-21T00:22:37 & 44.7812 & 6.7133 & 10.2 & 1.1 & Italian-French border \\
\hline 2018-08-21T01:31:42 & 44.4553 & 7.3338 & 10.9 & 0.6 & Roccabruna \\
\hline 2018-08-22T02:31:59 & 45.7887 & 7.4343 & 4.5 & 1.1 & Quart \\
\hline 2018-08-22T09:59:57 & 44.2657 & 7.4883 & 8.2 & 1.1 & Roaschia \\
\hline 2018-08-22T22:16:01 & 44.4025 & 7.0607 & 8.7 & 2.1 & Canosio \\
\hline 2018-08-23T12:26:07 & 44.6858 & 7.2413 & 11.2 & 0.9 & Paesana \\
\hline 2018-08-25T09:06:07 & 44.5365 & 6.9138 & 10.9 & 1.5 & Acceglio \\
\hline 2018-08-27T07:42:40 & 45.6678 & 7.6605 & 10.0 & 0.9 & S. Champ depraz \\
\hline 2018-08-27T20:29:22 & 44.3483 & 6.8402 & 10.2 & 1.1 & Italian-French border \\
\hline
\end{tabular}




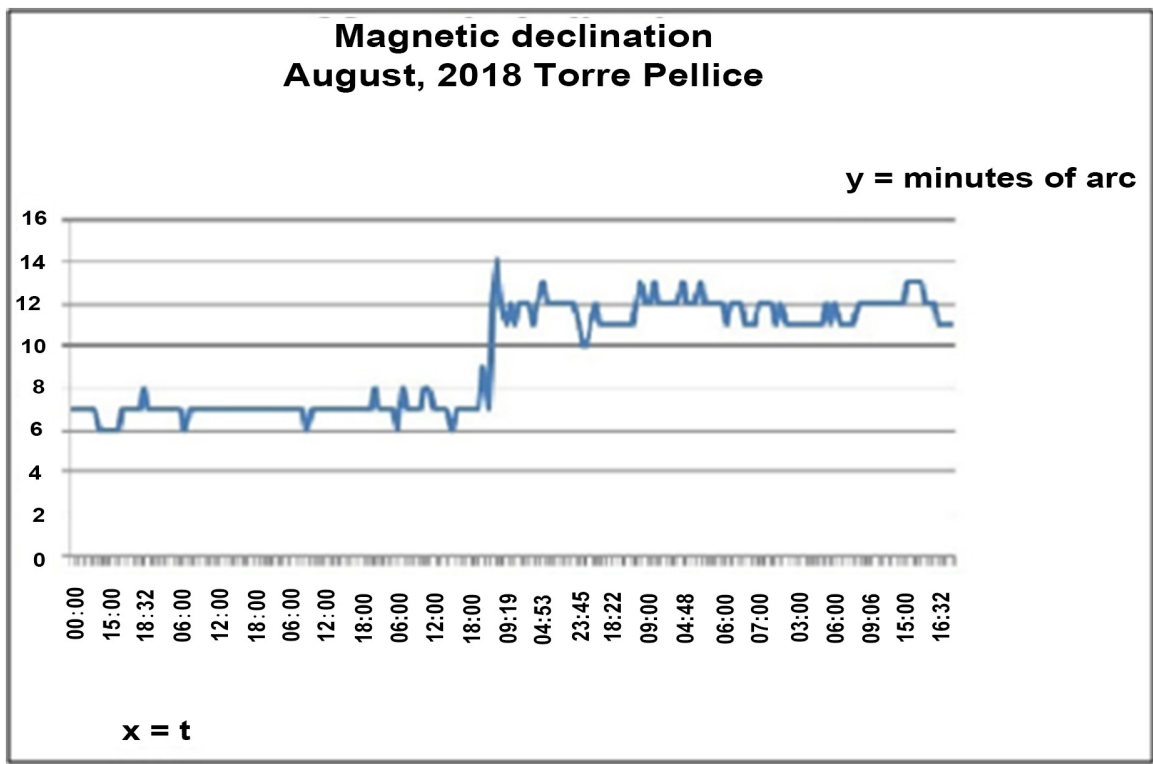

Graphic 1. $\mathrm{x}=\mathrm{t}$ local time.

\section{June 05-2009 magnetic declination toward West}

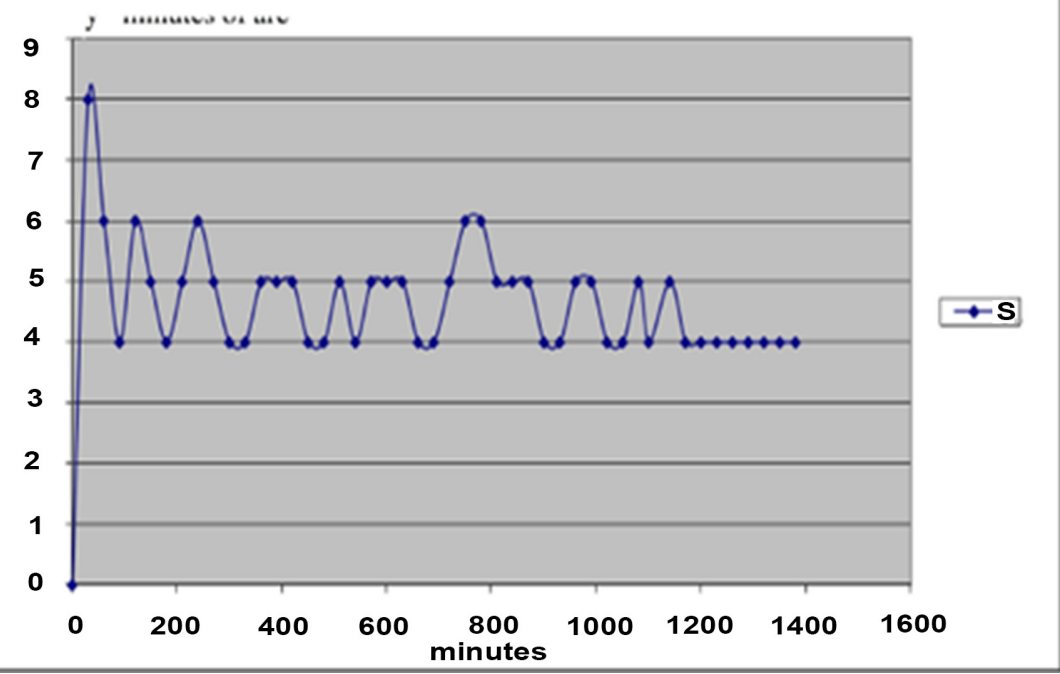

Graphic 2. Short $A_{1}$ and longer $A_{2}$.

Graphic 3 shows the variations of magnetic declination of phase $A_{1} 12$ hours before the earthquake with epicentrum at Roaschia, occurred on September 1, 2003. The values of irregular ascending peaks, typical of phase A1 and not of phase A2, can be seen.

When Phase B begins, the super group alpha dog continues the sequence 10 alone, enriches it with other sounds of the Pythagorean scale and creates a "song" with ever-detached sounds, of ternary rhythm, but with a slower agogic. This barking is very creative, similar to the howling of the alpha wolf, when it howls alone, on the other hand, when the ululates are in a group, they have 
magnetic declination $y=$ minutes of arc

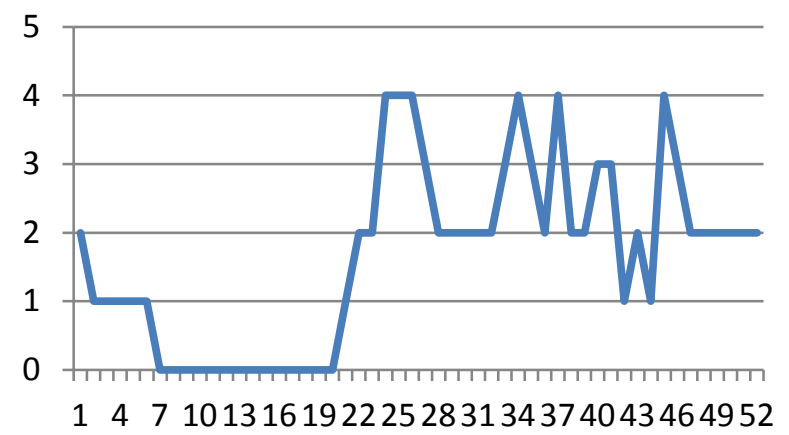

Graphic 3. Magnetic declination: Phase $A_{1}, 12$ hours before the seism occurred on Sept. 1, 2003 at 7:28:11 p.m., in Roaschia $(\mathrm{CN})$.

a very lively agogic. Therefore, there recognizably seems to be a growing weariness in the Phase B sequences of the alpha dog, since the intervals of silence between one sound and the other are always longer, while maintaining the ternary rhythm, in practice it sings with a "slowing down" of ever increasing music. On the other hand, the previous phases have excited them, and the alpha dog is more stressed than other dogs. These sequences are rich in associations of three, or four, then two descending sounds, interspersed with breaths and resumed above, then again three, then two then a single sound, which is finally repeated a few times, but the phoneme is very short. The last phonemes of a single "phono" are not $\mathbf{w}^{*} \mathbf{u}^{*}$ but $\mathbf{b}^{*}$, a typical phoneme of a calm dog in its territory.

So, the last vocalizations of $B$ can often be:

$$
\begin{aligned}
& 11] \rightarrow\left[G_{3}, F_{3}, E_{3}(b) \text {, pause, pause, } A_{3}(b), G_{3}\right. \text {, pause, } \\
& B_{3} \text {, pause, pause, } A_{3}(b), G_{3} \text {, pause, } \\
& A_{3}(b), G_{3}, B_{3}, \text { pause, pause, pause, } A_{3}(b), G_{3} \text { pause, } \\
& \left.B_{3} \text {, pause, pause, } B_{3} \text {, pause, } C_{4} \text {, pause, pause, pause }\right] \ldots
\end{aligned}
$$

The uppercase letters indicate the notes of the scale and the " $U \mathrm{t}$ " is indicated with the $\mathrm{A}$, while the lower apex 3 indicates the third octave of the piano, the ventral octave, the apex 4 indicates the fourth octave.

It is interesting to note that when the sound is a FA (F) followed by a SI (B), immediately afterwards, or after a few other sounds, the alpha dog emits a UT (C), almost to seek a spontaneous resolution of the tri-tone FA-SI (F-B), interval of the fourth excess. In medieval music this interval was called "diabolus in musica", difficult to sing for the singers, so much so that the Si "bequadro", or natural, was then lowered to B flat. The difficult intonation is due to a reluctance to take intervals that in the Pythagorean scale do not follow the pattern of the right ascendant wings, which turned into the lower octave become intervals of the correct fourth. All the excess intervals have the effect on humans and on dogs of reinvigorating attention, an example is the sirens of ambulances or the police, the two sounds are in relation to each other in excess ratio. When dogs howl at the ambulance sirens, they are extremely alert and frightened, since, on a seman- 
tic level, this range in their vocal language means danger. The dog howling, like the wolf howling, is an expression of a feeling of sadness and abandonment in view of danger. Dogs also moan when they hear bells, but even here the intervals are almost never a fourth or fifth, since the bells are often out of tune. In addition, bells have many harmonics and the ones highest in pitch are annoying since they are not consonants in relation to the fundamental sound; the dogs perceive them, given their extraordinary acoustic sensory apparatus.

We can resume in Table 17 body postures of the dogs personally observed, in relation to the three pre-seismic moments.

It is interesting to note the emotional situation of the alpha dog of the super group, the apparent sense of loneliness and sadness could be connected to the fact that in the pre-seismic phase B the birds and other animals of the wood are silent, even those of the farms. Also the declension anomaly that had destabilized the dogs ceased. It is not known if this return to normality of the declination and of the variations in intensity of the geomagnetic field brings the alpha dog to a relaxation after the previous state of stress, with consequent posture of the body similar to that assumed in depression.

\subsection{Local Micro-Seismicity and More Distant Earthquakes of High Magnitude: The Complex Synergy of Anomalies Destabilizes Dogs (and Not Only)}

Recent scientific literature has shown the relationship between seismic and volcanic activity with variations in natural electrical, magnetic and electromagnetic (EM) parameters. The magnitude of the pre-seismic variations is related to the intensity of the magnitudes of future earthquakes in preparation and to the distance of the places where such perturbations occur from the epicenters and also from the hypo-centers of the seismic nucleation zones. It should be noted that anomalies of behaviour and particular pre-seismic vocal sequences have always come true for local earthquakes, even of low magnitude. The difficulty is to discern which physical parameters of the anomalies influence the behaviour of dogs, in a pre-seismic phase both of local earthquakes of very low magnitude and of distant earthquakes of high magnitude, with a time interval of a few days. An interesting example of a complex synergy of anomalies can be the sequence of few local earthquakes within $90 \mathrm{~km}$ of SPSC and with very low magnitude that happened on May 16, 18, and 19, and the "earthquakes of Emilia" on May 20, 2012, which had the first shock of high magnitude $M L=5.9$, with the epicenter in Finale Emilia and depth of $7 \mathrm{~km}$. The earthquake was followed by others of high magnitude on May 29, with $\mathrm{ML}=5.8, \mathrm{ML}=5.3, \mathrm{ML}=5.2$. The swarm lasted for months. Finale Emilia is $320 \mathrm{~km}$ from SPSC, but the nucleation of a large magnitude earthquake inevitably leads to the presence of precursors on a larger area.

While Table 18 shows examples of local earthquakes, Table 19 shows the related phases $A_{1}$ and $A_{2}$ and $B$ and magnetic declination, with the vocal sequences of the dogs associated with them. 
Table 17. Pre-seismic sequences and body postures.

\begin{tabular}{|c|c|c|c|}
\hline Pre-seismic phases & $\begin{array}{c}\text { Dogs } \\
\text { personally } \\
\text { observed }\end{array}$ & Vocal sequences & Body Postures \\
\hline $\mathrm{A}_{1}$ & $1,2,3,4,5,6,7$ & Sequence n. 8 & $\begin{array}{l}\text { Pupils dilated ears back, open mouth } \\
\text { angles, tail aligned to the vertebral } \\
\text { column, posture often raised on hind } \\
\text { legs with pirouettes of the body, high } \\
\text { snout at the top. Fear and surprise. }\end{array}$ \\
\hline $\mathrm{A}_{2}$ & $1,2,3,4,5,6,7$ & Sequences n. 9,10 & $\begin{array}{l}\text { Pupils dilated, ears back, nose } \\
\text { wrinkled, lips slightly curled, corner } \\
\text { of mouth pulled back, hackles raised, } \\
\text { tail tucked, body lowered. } \\
\text { Aggression }\end{array}$ \\
\hline B & 27 & $\begin{array}{l}\text { Sequences n. } 11 \text { and } \\
\text { similar }\end{array}$ & $\begin{array}{l}\text { Lowered head, generally seated body, } \\
\text { curved spine, smooth hair, low tail, } \\
\text { dog looking towards different } \\
\text { directions. Sadness and solitude. }\end{array}$ \\
\hline
\end{tabular}

Table 18. Local earthquakes.

\begin{tabular}{cccccc}
\hline Time UTC & Latitude & Longitude & Depth km & ML & Event location \\
\hline 2012-05-16T18:16:14 & 44.4923 & 6.7503 & - & 1.2 & Italian-French border \\
2012-05-16T20:27:31 & 44.8105 & 7.2227 & 14 & 1.3 & Torre Pellice (TO) \\
2012-05-18T07:30:52. & 44.2842 & 7.4518 & 2 & 0.7 & Roaschia (CN) \\
2012-05-19T02:32:16 & 44.1402 & 7.8833 & 2 & 0.9 & Ormea (CN) \\
2012-05-19T02:48:28 & 44.1207 & 7.8883 & 4 & 1.4 & Ormea (CN) \\
\hline
\end{tabular}

Table 19. Phase $A_{1}, A_{2}$ and $B$ and magnetic declination.

\begin{tabular}{|c|c|c|c|c|c|}
\hline UTC time & $\begin{array}{l}\text { Phase } A_{1} \\
\text { UTC time }\end{array}$ & $\begin{array}{l}\text { Phase } \mathrm{A}_{2} \\
\text { UTC time }\end{array}$ & $\begin{array}{l}\text { Phase B } \\
\text { vocal sequence of } \\
\text { alpha dog }\end{array}$ & $\begin{array}{c}\text { Magnetic } \\
\text { declination } \\
\text { initial } \mathrm{A}_{1} \\
\text { minutes of arc }\end{array}$ & $\begin{array}{c}\text { Dog's vocal } \\
\text { sequences } \\
\text { of } A_{1}\end{array}$ \\
\hline $\begin{array}{l}\text { 2012-05-16 } \\
\text { T18:16:14 }\end{array}$ & h.11:32 & // & // & $\delta=0.5 \mathrm{E}$ & 8] \\
\hline $\begin{array}{l}\text { 2012-05-16 } \\
\text { T20:27:31 }\end{array}$ & h. 13:08 & // & $\begin{array}{c}\text { h.17:15 } \\
\text { sequence 10],11] } \\
\text { and similar to 11] }\end{array}$ & $\delta=1 \mathrm{E}$ & 8] \\
\hline $\begin{array}{l}\text { 2012-05-18 } \\
\text { T07:30:52 }\end{array}$ & h. $0: 23$ & // & $\begin{array}{l}\text { h. } 01: 15 \\
\text { sequence similar } \\
\text { to } 11]\end{array}$ & $\delta=1 \mathrm{E}$ & 8] \\
\hline $\begin{array}{c}2012-05-19 \\
\text { T02:32:16 } \\
2012-05-19 \\
\text { T02:48:28 }\end{array}$ & $\begin{array}{l}\text { h.21:15 on } \\
\text { May } 18\end{array}$ & // & $\begin{array}{c}\text { h. } 01: 15 \\
\text { sequencesimilar to 11] }\end{array}$ & $\delta=1 \mathrm{E}$ & 8] \\
\hline
\end{tabular}

Table 20 again shows the phase $A_{1}, A_{2}, B$, the magnetic declination and the related vocal sequences of the dogs, but related to the earthquake of Finale Emilia in 2012. 
Table 20. Emilia's earthquake occurred on 2012-05-20 at 02:03:50, UTC time.

\begin{tabular}{|c|c|c|c|c|}
\hline $\begin{array}{l}\text { Initial Phase } A_{1} \\
\text { UTC time }\end{array}$ & $\begin{array}{c}\text { Dog's vocal sequence } \\
\text { in Phase } \mathrm{A}_{1} \\
\text { Initial Magnetic } \\
\text { Declination } \\
\delta \text { in minutes of arc }\end{array}$ & $\begin{array}{l}\text { Phase } A_{2} \\
\text { UTC time }\end{array}$ & $\begin{array}{c}\text { Dog's vocal sequence in } \\
\text { Phase } A_{2}\end{array}$ & $\begin{array}{c}\text { Phase B } \\
\text { vocal sequence of alpha dog } \\
\text { Stable position of the compass needle: } \\
\text { magnetic Declination } \delta \text { in minutes of arc }\end{array}$ \\
\hline h. $10: 20-17: 15$ on Mai, 19 & $\begin{array}{c}8] \\
\delta=24 \mathrm{E}\end{array}$ & $\begin{array}{l}\text { h. } 17: 15 \text { on May } 19 \\
\text { to h.0:38 of May } 20\end{array}$ & $\begin{array}{l}9] \\
\text { at the end of } \mathrm{A}_{2} \text { also 10] }\end{array}$ & $\begin{array}{l}\text { h. } 01: 13 \text { on May, } 20 \\
\text { 10], 11] and similar }\end{array}$ \\
\hline
\end{tabular}

The aforementioned local earthquakes in Piedmont, some of which, of very low magnitude, are only instrumentally detected, are expression of a crustal deformation.

They were preceded by weak magnetic anomalies, punctually perceived by dogs and other magneto-sensitive animals, such as birds. It is interesting to note the concomitance of the radon emission peak two days before the earthquake in Finale Emilia, as can be seen in Graphic 4. The high values of radon emitted in a few days immediately damage small animals such as spiders, insects, snails or amphibians or reptiles; the dogs however, often raise their snouts to smell the strongly ionized air [4].

Other anomalies were recorded during that period.

The ELF and VLF detector installed by the physicist Cristiano Fidani on the roof of SPSC has recorded anomalies before both local earthquakes and the earthquake in Emilia. A few examples of ELF and VLF monitoring will be commented regarding the responses of dogs [43].

Figures 5-11 represent some examples taken from the continuous monitoring of ELF and VLF transmitted by the processing performed by the "Spectrum Lab" installed in SPSC for ELF and VLF anomalies, recorded on 18, 19 and 20 May 2012.

It is also useful to compare the weather data recorded in Pinerolo, at a distance of $10.58 \mathrm{~km}$ from SPSC, in the week of the earthquake of Finale Emilia, as shown in Table 21.

As can be seen from the table on the weather, the anomalies in temperature, gusts of wind, and rain characterized Piedmont in those days, and the images obtained with the Spectrum Lab show the effects of thunderstorms. Dogs, as well as cats, perceive weather variations well in advance, even 8 - 10 hours in advance. They respond to them not with particular vocalizations, but with postures typical of fear and with a search for a hiding place, especially from the wind: on that occasion, my dogs had dug a large den in the wooded part of the garden; even though they could hide in the house, they preferred their burrow in the earth, dug under branches. This method of wind protection had been taught to them by the bitch, mother wolf's daughter. Before the arrival of the wind, other dogs observed by me or their owners had sought refuge in their kennel, or those who could, inside the stables. 


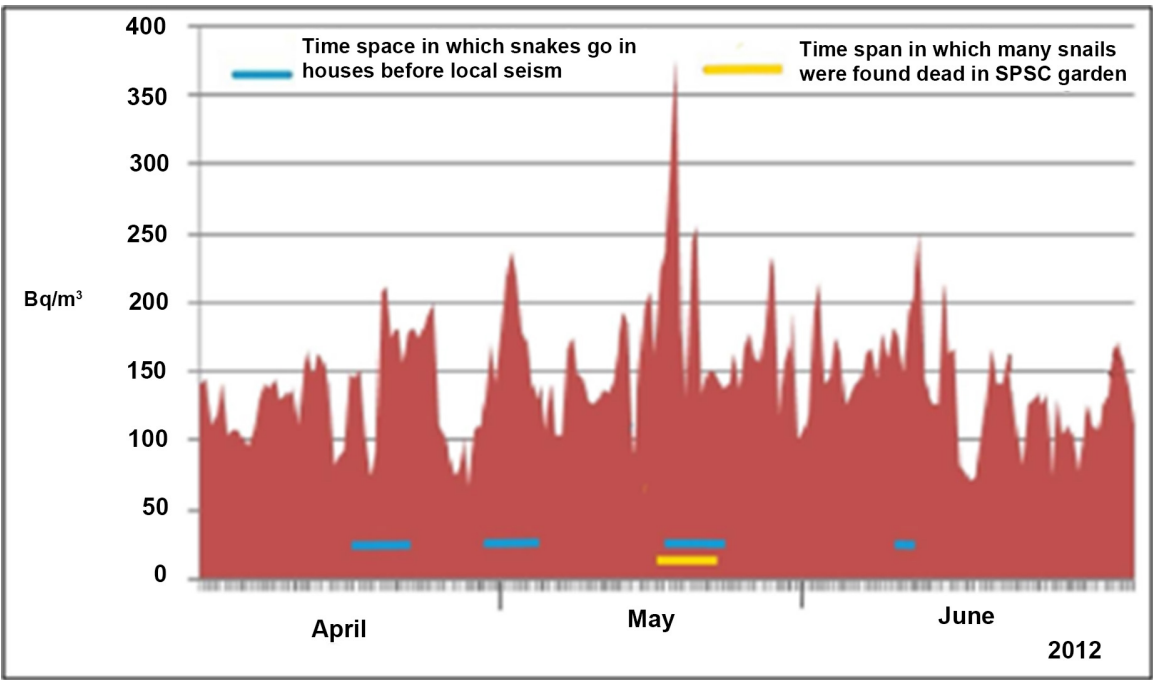

Graphic 4. Radon 222 emission monitored into SPSC.

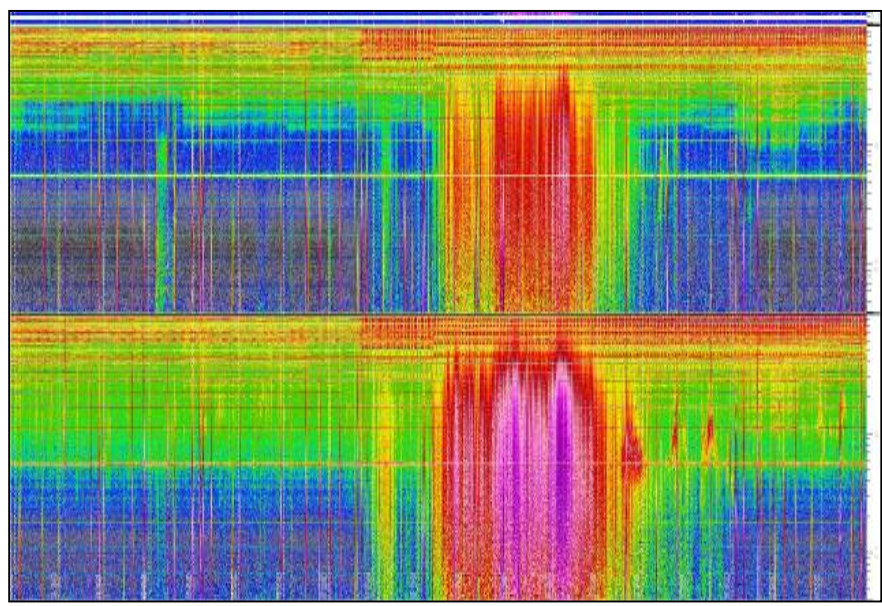

Figure 5. ELF recorded near SPSC on 18/05/2012, LT 8:00 am. Cristiano Fidani's monitoring.

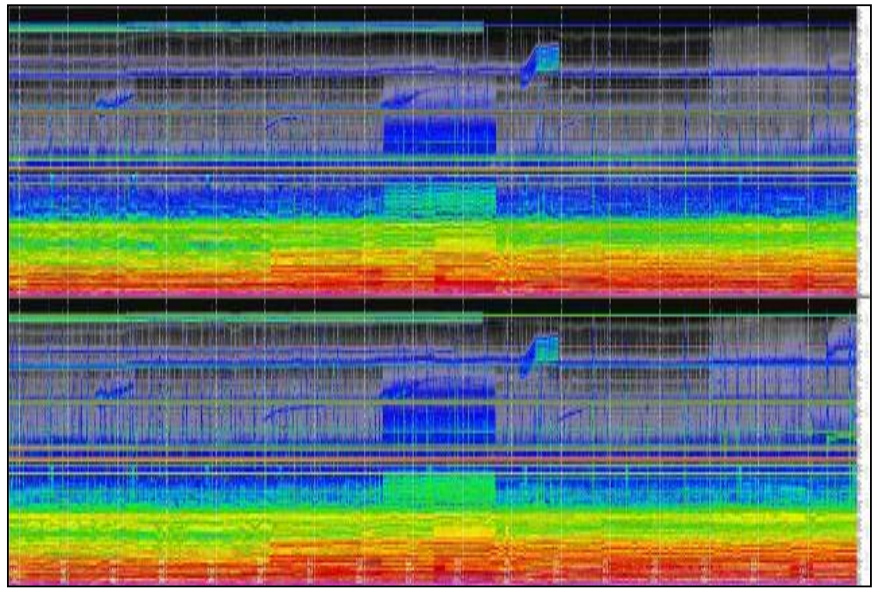

Figure 6. VLF recorded near SPSC on 2012-05-18, LT 8:00 am. Cristiano Fidani's monitoring. 


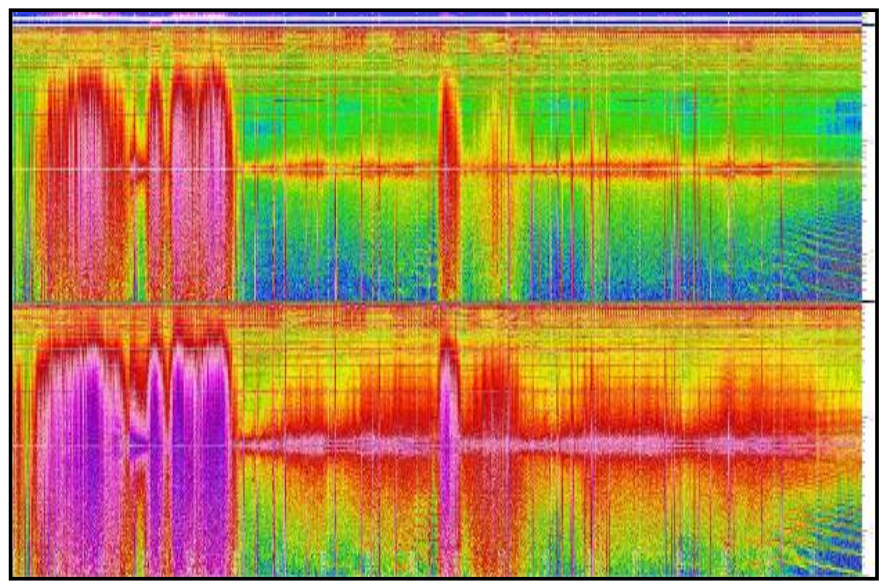

Figure 7. ELF recorded near SPSC on 2012-05-18, LT 10.40 am. Cristiano Fidani's monitoring.

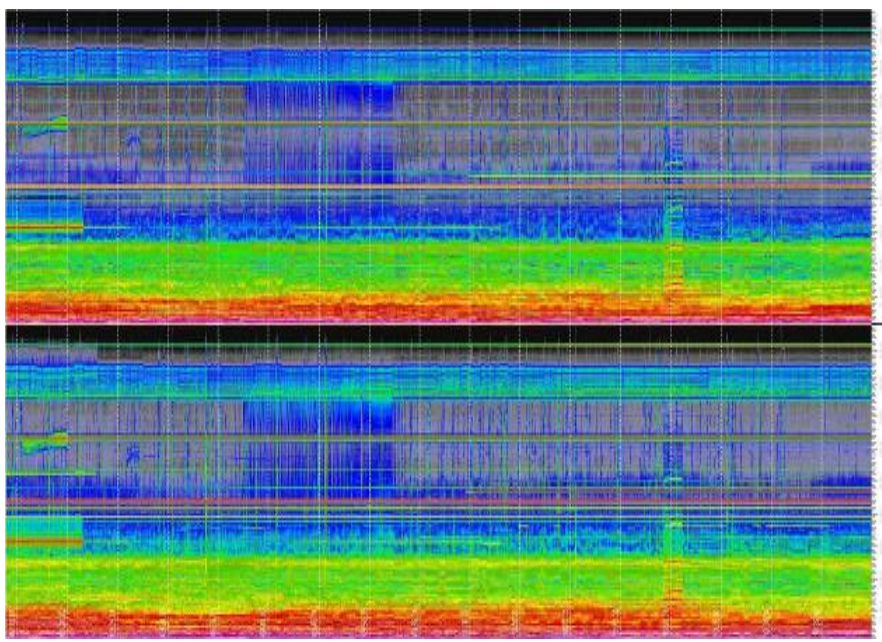

Figure 8. VLF recorded near SPSC on 2012-05-18, LT 10.40. am. Cristiano Fidani's monitoring.

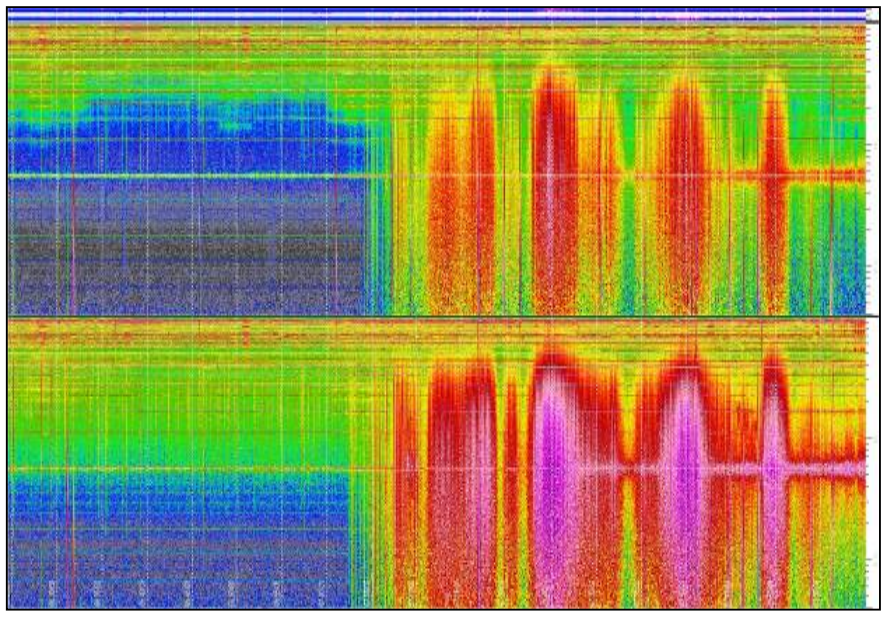

Figure 9. ELF recorded near SPSC on 2012-05-19, LT 08.00 am. Cristiano Fidani's monitoring. 


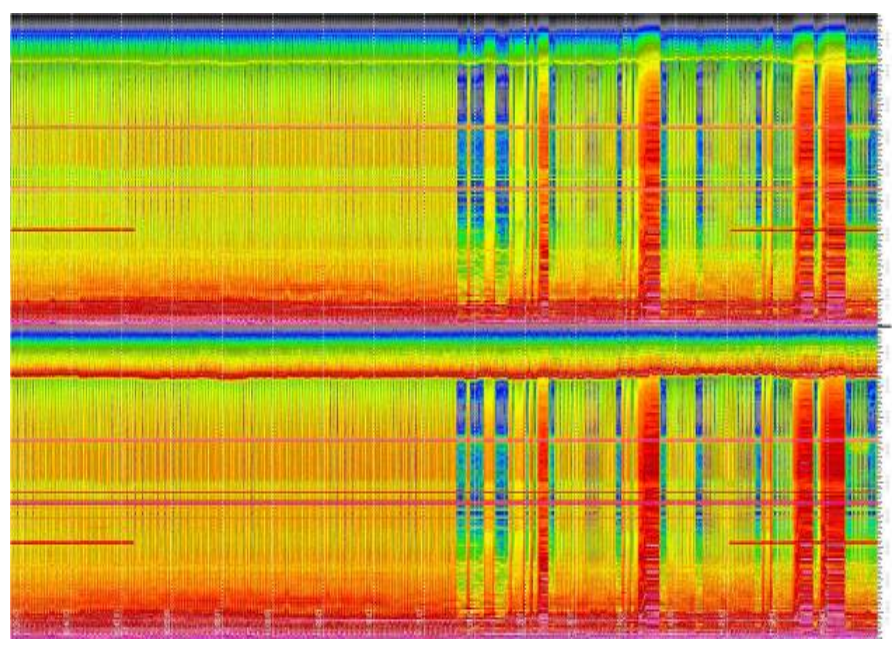

Figure 10. VLF recorded near SPSC on 2012-05-20, LT $12.00 \mathrm{am}$. Cristiano Fidani's monitoring.

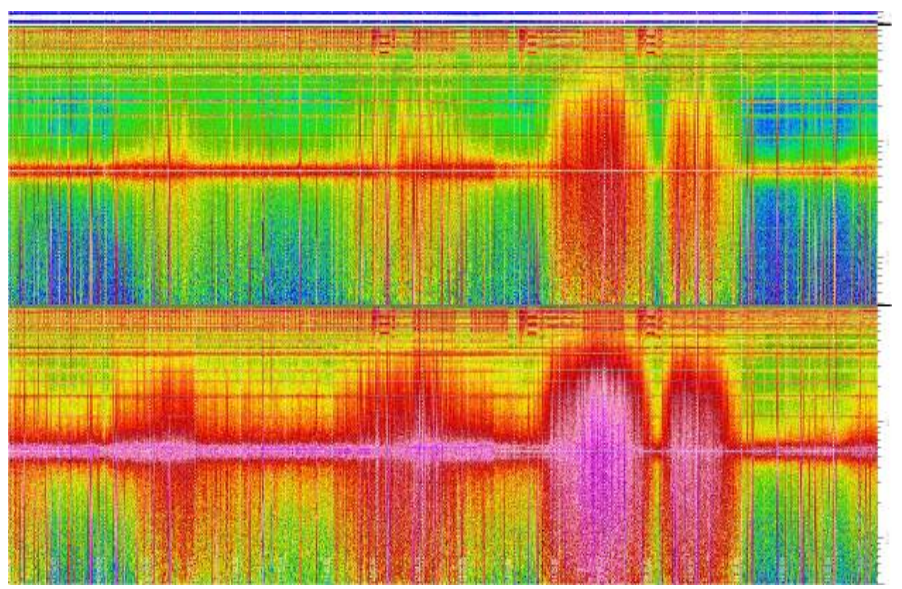

Figure 11. ELF recorded near SPSC on 2012-05-20, LT 14.40 pm.

\section{Brontides, Natural Explosive Noises and Dogs' Vocal Sequences}

The area where the observation Center of the seismic precursors is located is affected by an interesting and little known phenomenology of natural noises called "brontides", or "mistpouffers".

If these natural sounds occur in the air they can be confused with gunshots, they are very short and have no echo, but a better sound analysis of them leads us to glimpse their timbre which is similar to the snap of the "percussive wood" musical instruments.

If they are produced in the subsoil, they can take on different tonal characteristics and sound duration: sometimes these noises are similar to underground closures of large doors, with a very short duration, sometimes they resemble drum rolls with local tremors and with a longer time duration, but beyond the second one, sometimes in subterranean landslides from the muffled noise, the noise then produced is longer, with a local tremor [44]. 
Table 21. Weather data from Pinerolo, 2012-05-17/24.

\begin{tabular}{cccccccc}
\hline Day & T Average & $\mathrm{T}$ min & T max & Rainfall & Humidity & Wind Max & $\begin{array}{c}\text { Phenomena/ } \\
\text { gusts }\end{array}$ \\
\hline 13 & $16^{\circ} \mathrm{C}$ & $12^{\circ} \mathrm{C}$ & $21^{\circ} \mathrm{C}$ & $\mathrm{n} / \mathrm{d}$ & $72 \%$ & $33 \mathrm{~km} / \mathrm{h}$ & $46 \mathrm{~km} / \mathrm{h}$ \\
14 & $15^{\circ} \mathrm{C}$ & $11^{\circ} \mathrm{C}$ & $20^{\circ} \mathrm{C}$ & - & $58 \%$ & $15 \mathrm{~km} / \mathrm{h}$ & - \\
15 & $16^{\circ} \mathrm{C}$ & $9{ }^{\circ} \mathrm{C}$ & $22^{\circ} \mathrm{C}$ & - & $50 \%$ & $39 \mathrm{~km} / \mathrm{h}$ & $63 \mathrm{~km} / \mathrm{h}$ \\
16 & $16^{\circ} \mathrm{C}$ & $12{ }^{\circ} \mathrm{C}$ & $19^{\circ} \mathrm{C}$ & - & $18 \%$ & $52 \mathrm{~km} / \mathrm{h}$ & $76 \mathrm{~km} / \mathrm{h}$ \\
17 & $11{ }^{\circ} \mathrm{C}$ & $6^{\circ} \mathrm{C}$ & $18^{\circ} \mathrm{C}$ & - & $42 \%$ & $20 \mathrm{~km} / \mathrm{h}$ & $\mathrm{no}$ \\
18 & $15^{\circ} \mathrm{C}$ & $12^{\circ} \mathrm{C}$ & $19^{\circ} \mathrm{C}$ & $\mathrm{n} / \mathrm{d}$ & $49 \%$ & $19 \mathrm{~km} / \mathrm{h}$ & 88 \\
19 & $14^{\circ} \mathrm{C}$ & $10^{\circ} \mathrm{C}$ & $18^{\circ} \mathrm{C}$ & $\mathrm{n} / \mathrm{d}$ & $68 \%$ & $11 \mathrm{~km} / \mathrm{h}$ & 88 \\
20 & $13^{\circ} \mathrm{C}$ & $11^{\circ} \mathrm{C}$ & $16^{\circ} \mathrm{C}$ & $\mathrm{n} / \mathrm{d}$ & $82 \%$ & $15 \mathrm{~km} / \mathrm{h}$ & 88 \\
21 & $11{ }^{\circ} \mathrm{C}$ & $10^{\circ} \mathrm{C}$ & $18^{\circ} \mathrm{C}$ & $\mathrm{n} / \mathrm{d}$ & $88 \%$ & $26 \mathrm{~km} / \mathrm{h}$ & 88 \\
22 & $15^{\circ} \mathrm{C}$ & $11^{\circ} \mathrm{C}$ & $20^{\circ} \mathrm{C}$ & $\mathrm{n} / \mathrm{d}$ & $70 \%$ & $20 \mathrm{~km} / \mathrm{h}$ & 88 \\
\hline
\end{tabular}

Sometimes brontides can be confused with explosions in the Barge area that take place in the quarries of the "Luserna stone" (gneiss) in Rorà, less than 10 $\mathrm{km}$ from the Study Center. But in this case, the tonal characteristics and the frequencies of the explosions are different from those of the brontides, moreover, allowing important observation for the contribution that the dogs give to discern events: for the anthropogenic explosions, the dogs bark later, for the brontides with local tremors, dogs give the alarm first, usually 40 " before, as in phase $\mathrm{C}$ of seismic events, but in this case they perceive the p. waves [45].

Sometimes when the natural explosions take place in the air, sulphureous smells are perceived.

These sounds are also heard in certain seismic regions especially along seacoasts or over lakes, or near quicksand. Often brontides are precursors of earthquakes of high magnitude, but not for all seismic activity.

Sometimes, during a high emission of Radon $\left(\mathrm{Rn}^{222}\right)$, there are also numerous brontides, as we can see in Table 17. This event occurred one day before a local earthquake, with magnitude $\mathrm{ML}=2.6$, a depth of $10 \mathrm{~km}$ and epicentrum distance of less than $10 \mathrm{~km}$, on the Italian-French border. On this occasion, both high Radon emission and brontides were seismic precursors, with a temporal and spatial forecast.

The seismic event that occurred on 03/12/2013 had a short-term temporal forecast, for the rapid decrease of radon emission, for the anomalies of geo-magnetic field intensity, and for the moaning of the alpha dog 4 hours before the seismic shock. Regarding the spatial prediction, however, I could only believe on December 2 that the future earthquake could have occurred within a radius of 15 $20 \mathrm{~km}$ because the magnetic declination anomaly had only been 1 degree to the west.

During the day of December 2, over 15 brontides were perceived, all announced 40 " in advance by the dogs with the sequence 6 : 


$$
6] \rightarrow\left[\mathrm{w}^{*} \mathrm{o}^{*} \cup \mathbf{a}^{*}, \mathrm{w}^{*} \mathrm{o}^{*} \cup \mathbf{a}^{*}, \mathrm{w}^{*} \mathbf{o}^{*} \cup \mathbf{a}^{*}\right]
$$

The extremely refined auditory perception of the dogs allows them to hear the infra sounds and therefore to perceive all those elastic deformations of the subsoil.

Graphic 5 shows an important ascending peak, before the earthquake of Finale Emilia, of the continuous monitoring of radon registered in SPSC. During this anomalous increase there were numerous brontides.

The fact that the dogs observed and even those further away (those on the mountain in front, to the south, closer to the epicentrum of the earthquake in the nucleation) had all issued sequences like the number 6, with intervals between the sounds of the right quarter and not excessively, always with 40 " in advance, makes me think that on that day, every brontide had some local tremors, not registered as earthquakes by INGV stations.

Furthermore, those brontides had a type of noise like underground drums or small landslides, some of them gave me the same perception of tremor under my feet when a thunderbolt strikes less than $1 \mathrm{~km}$ from me.

From the infra-sound monitoring, the observation of the sound spectra of the brontides in the intervals of frequencies between 0.1 and $30 \mathrm{~Hz}$ provides useful elements to understand at least the modes of sound transmission, however, without managing to formulate the precise causes that originate such natural noises.

The following image n. 12 shows a Spectrum Lab elaboration of different underground and air rumbles, with images referring only to infrasound intervals with frequencies between $0.1-30 \mathrm{~Hz}$, so the human audible spectrum is missing.

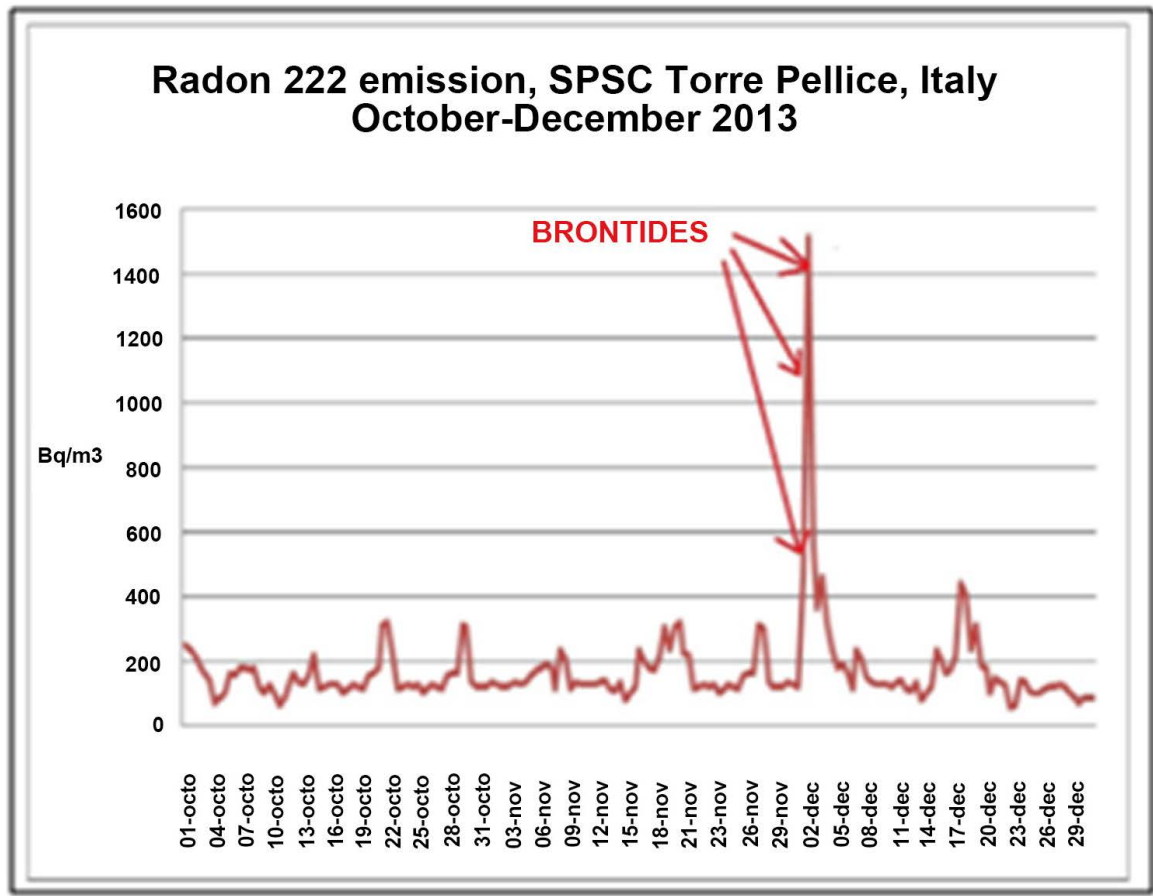

Graphic 5. Radon $\mathrm{Rn}^{222}$ emission and brontides. 
If these natural sounds occur in the air (case D), they are very short, like the sound of gunshots, generally one or two explosions. Dogs bark after the explosion. If only one explosion occurs, the dogs quickly overcome their surprise, identify the direction of origin of the noises, and issue the interrogative sequence n. 5; if the outbreaks are two or more, then they "warn the noises" to stay away, as if they were intruders, so the sequence is n. 6 , repeated until there are brontides, with increasing aggression, depending on the number of noises.

In the case of underground noises without local tremors, the response of the dogs is similar to the brontides in the air in the case of noises similar to large air bubbles that go up a column of water; if the sound source is very close to home, dogs growl looking at the ground.

In the case of underground noises with local tremors, the sounds generally follow the tremor, and are like subdued roars, like drum rolls, like noises of small landslides, expression of a local seismicity with extremely low magnitude, I would say more a destabilization of local fans, due to moving water masses. This is my hypothesis, which I have inferred from the following geological technical report by the Municipality of Torre Pellice. In this case, the dogs emitted the typical sequence n.6, the one also emitted for phase $C$ that precedes the earthquakes, 40 seconds in advance of when the brontides were heard.

There are many hypotheses about the origin of the brontides, one of which is that changes in the flow of water in canals and underground caves can induce these noises, sometimes followed by earth tremors, sometimes followed by earthquakes of higher magnitude. In any case, to better understand its origin, it is useful to consider the local geological morphology. With reference to the geological map compiled by the surveys of the Municipality of Torre Pellice and the ARPA of the Piedmont Region, the following can be summarized thus:

With regard to the rocky substrate, in the area under investigation, the Crystalline Pretriassic Complex consists essentially of two large groups: gneiss and micaschists. The presence of gneiss is indicative of regional dynamo-thermal metamorphism of original rocks (protoliths) of sialic composition, or rich in quartz and feldspar and less than iron-magnesium silicates. The metamorphic process is formed at great depth, due to the high values of pressure and temperature, in areas of convergence of two plates during the phase of subduction and then of continental collision, from which the mountain ranges originate.

At a distance of less than $1 \mathrm{~km}$ from the SPSC Center, it is present as a rock substratum two-handed Gneiss, of which the sub-vertical walls of Mt Castellus and "Bars d'la Taiola" are formed. They are made of light-colour, medium-sized gneiss, not very schistose and of massive appearance, and are composed of potassic feldspar, plagioclase, white mica, biotite, and quartz.

Regarding the quaternary coverage in the territory of Torre Pellice, essentially it consists of alluvial products, glacial deposits, gravity deposits, and eluvio-colluvial and debris-eluvial products. The Study Center lies on the right bank of the Biglione river, facing south, on a large conoid geomorphology of the Rio Biglione, a conoid that originates upstream from Borgata Servera and extends towards the 
east and south, to form an inclined plane with regular slope of less than $15^{\circ}$. "On the whole area under consideration, there are no outcrops of the rocky substratum, not even at the base of the high slope of the Pellice, so it can be assumed that the power of the depositional body is, at least in the centrum-front sector, over 30 meters, as was also found in the survey carried out on the right bank alongside the bridge on the $S P$ ( $n .5$ in the "Paper of litho-technical and hydro-geological characteristics") in which, after $40 \mathrm{~m}$ of coarse deposits of conoid, it was not the substratum that was found but silty, clayey gray lake deposits reaching a depth of up to 91 meters" [46]. The body of the fan is home to an extended and continuous aquifer that is leveled at considerable depth.

Since during the long observation of the behaviour of my dogs I have repeatedly seen my dogs look on the ground and growl, without at that time I could hear some noises, I thought that in addition to the brontides I heard there were also underground frequencies in intervals of infrasound, so I started to study them with an instrument from 2013 to 2016.

The infra-sounds were monitored by an "Aetech Infra-sounds Monitor", with an USB range of measures $0-20 \mathrm{~Hz}$, while the VCO (Voltage Controlled Oscillator) range of measures was $0-35 \mathrm{~Hz}$. So the Spectrum Lab elaboration, a chromed-graphic elaboration, showed me only the elaboration of the infrasound included in the described range, while the noises perceived by me are obviously in the audible frequencies for the human being. So I was able to observe in real time, according to the monitor, the graphic configuration in the infrasound spectrum of underground sounds of brontides or brontides in the air, verifying at the same time whether there were underground landslides or small local tremors, so to be able to read the different geometric shapes of the Spectrum Lab processing.

Figure 12 shows different elaborations of the infra-sound Spectrum Lab, in relation to brontides with or without tremors.

If we look at Figure 12, we can see that the geometric shapes of the sound spectrum are coloured, the colour refers to the intensity of the sound measured in decibels, so if we indicate with "i" the intensity of the harmonics of the sounds and their fundamental, we have the following:

$$
\begin{array}{ll}
\text { h }] \rightarrow \text { dark blue } & -58 \mathrm{~dB} \leq \mathrm{i}<-54 \mathrm{~dB} \\
\text { i }] \rightarrow \text { blue } & -54 \mathrm{~dB} \leq \mathrm{i}<-50 \mathrm{~dB} \\
\text { j } \rightarrow \rightarrow \text { light blue } & -50 \mathrm{~dB} \leq \mathrm{i}<-46 \mathrm{~dB} \\
\mathrm{k}] \rightarrow \text { green/blue } & -46 \mathrm{~dB} \leq \mathrm{i}<-42 \mathrm{~dB} \\
\text { l } \rightarrow \rightarrow \text { red } & -42 \mathrm{~dB} \leq \mathrm{i}<-38 \mathrm{~dB} \\
\text { m }] \rightarrow \text { red peony } & -38 \mathrm{~dB} \leq \mathrm{i}<-34 \mathrm{~dB} \\
\text { n } \rightarrow \rightarrow \text { orange } & -34 \mathrm{~dB} \leq \mathrm{i}<-30 \mathrm{~dB} \\
\text { o] } \rightarrow \text { yellow } & -30 \mathrm{~dB} \leq \mathrm{i}<-26 \mathrm{~dB}
\end{array}
$$

If we look at the geometric shapes, we can see that there is continuous and discrete overlapping of forms of Cassinian and Cartesian ovals (case A, B, C, D). In the absence of local tremors the isolated forms prevail and with non-close 


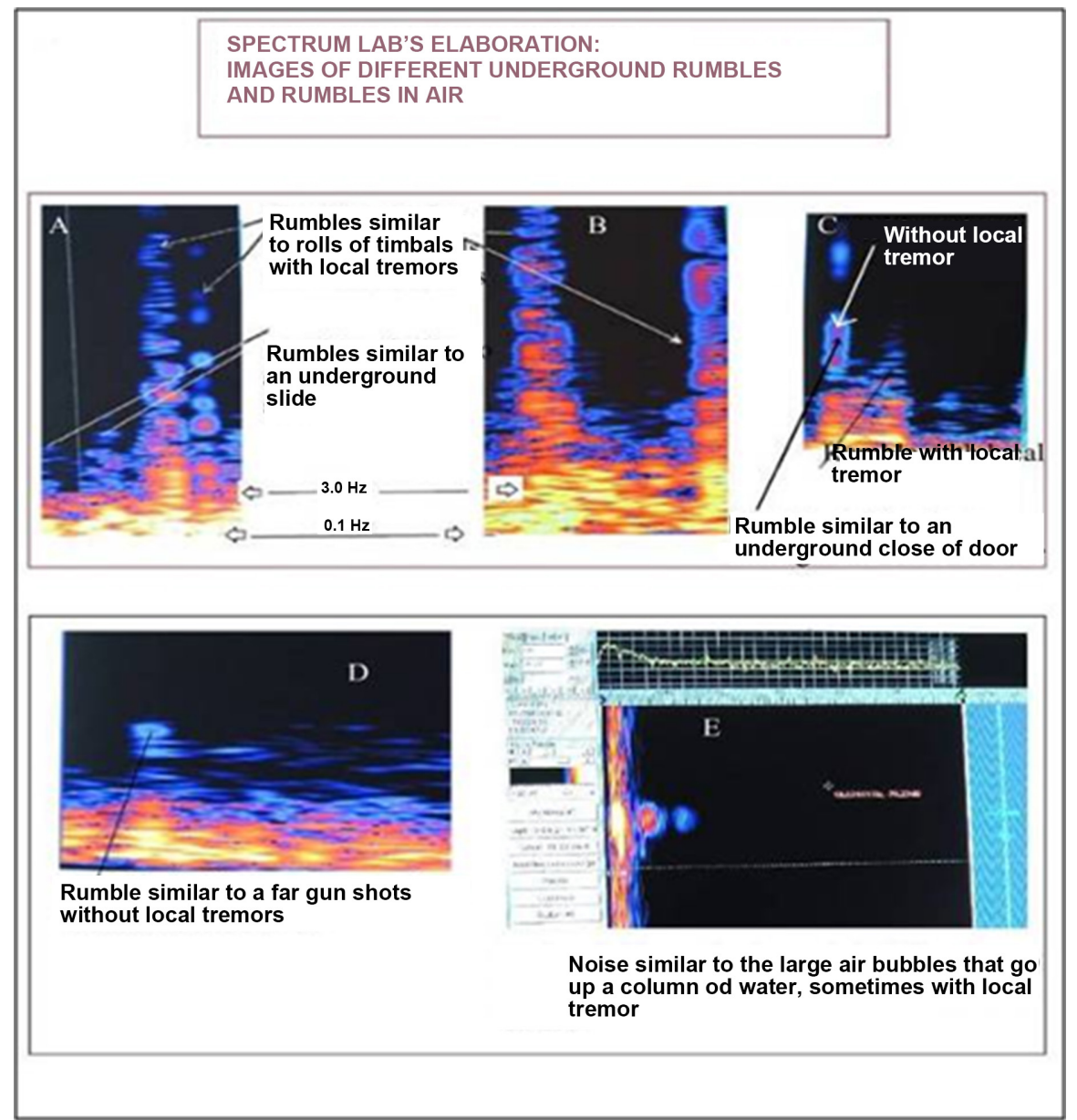

Figure 12. Underground rumbles and rumbles in air.

overlaps of the geometric shapes, generally 2 arches with a Saracen or Moorish profile, having the same axis in common, and therefore opposite (case E).

When the brontide follows a local tremor, the shapes of Cartesian ovals prevail among the oval figures in the elaboration of the Spectrum Lab and the noise is similar to an underground landslide.

\section{Conclusions}

The professional attitude to listen and analyze the sounds, even simultaneously emitted from multiple sources, has allowed me to highlight how dogs can communicate with each other with identical vocalizations over time to similar situations, experienced within a group or an enlarged group. The need for distance communication, no longer linked to body posture, has developed over the centuries a dog's ability to form a complex vocal language based on rhythmic and intoned sequences. This modality of communication best responds to the need for long-distance understanding and storing a "canine cultural" baggage, which takes on a semantic character. This character is confirmed by the testimonies of some owners of dogs, inhabitants in Pinerolo, S. Secondo, Villar Pellice, Rorà and Bobbio, in areas not inhabited, some of them have tried to sing the se- 
quences descendants of the alpha dogs of their villages, others called me when there was a particular sequence of the alpha dog, felt then by me at the same time the same sequence for rhythmic and for intonation character emitted near the Center by our alpha dog. This happened in phase $\mathrm{A}_{2}$ of seismic nucleations.

Having been able to identify particular semantic sequences in the variegated vocal language of dogs, all based on the fundamental functional needs of true interrogative, vocative, intimidating, imperative and communicative phrases of events of close interest to the group, created the necessary condition to attribute to certain sequences predictive value for earthquakes or for local hydro-geological instabilities.

Another necessary condition for a good seismic forecast [47] [48] [49] [50] is the continuous monitoring of possibly many physical parameters to identify the anomalies, to which to associate the particular vocal sequences of dogs.

\section{Acknowledgements}

Miriam Aimar, Laura Culver, Cristiano Fidani, Giovanni Martinelli, Lisa Pierotti, Anna Riggio, Alessandro Vaio, Grazia Vaio, Andrea Viotto, the owners of dogs, the dogs.

\section{Conflicts of Interest}

The author declares no conflicts of interest regarding the publication of this paper.

\section{References}

[1] De Liso, G. (2010) Caratterizzazione del territorio nell'individuazione dei precursori sismici locali nel Piemonte Occidentale. Atti del $29^{\circ}$ Convegno Nazionale GNGTS, Trieste, 14-17 Novembre 2010, 208-211.

[2] De Liso, G. and Fidani, C. (2014) Seismic Precursory Phenomenology in Unusual Animal Behaviour in Val Pellice, Western Piedmont, in Comparison with Anomalies of Some Physical Parameters. Open Journal of Earthquake Research (OJER), 3, 30-42. https://doi.org/10.4236/ojer.2014.31005

[3] De Liso, G., Fidani, C. and Viotto, A. (2013) Multiple-Parameter and Seismic Precursory Phenomenology in Unusual Animal Behaviour in Western Piedmont. Atti del $32^{\circ}$ Convegno GNGTS, Trieste, 19-21 Novembre 2013, 60-66.

[4] De Liso, G., Fidani, C. and Viotto, A. (2013) Unusual Animal Behaviour before Earthquakes and Multiple Parameter Monitoring in Western Piedmont. Animals, 3, 14-25.

[5] Boschi, E., Ferrari, G., Gasperini, P., Guidoboni, E. and Valensise, G. (1995) Catalogo dei Forti Terremoti in Italia dal 461 a.C. al 1980. ING-SGA, Bologna.

[6] Garola, L. (2012) Descrizione del Tremuoto nelle valli di Luserna, San Martino e Circondario di Pinerolo, Occorso li 2 Aprile 1808 con suoi Funesti Effetti d'Esso. Bollettino della Società Storica Pinerolese, III Serie, Anno XXIX. Pinerolo 2012, 178-189.

[7] Reycend, J.J. (1908) Lettre d'un Ancien Militaire à un Dame de Turin. Corrispondence Vaudoise, Turin, 1908, 13-14. 
[8] De Liso, G., Lattarulo, F. and Viotto, A. (2011) Piezonuclearità, Piezoelettricità e Piezomagnetismo delle Rocce di Gneiss e caratterizzazione territoriale della fenomenologia sismica nel Piemonte occidentale, nuove prospettive di studi. Atti del $30^{\circ}$ Convegno Nazionale GNGTS, 14-17 Novembre, 2011, Trieste, 603-604.

[9] Fidani, C. (2013) Biological Anomalies before the 2009 L'Aquila Earthquake. Animals, 3, 693-721. https://doi.org/10.3390/ani3030693

[10] Rikitake, T. (1978) Biosystem Behaviour as an Earthquake Precursor. Tectonophysics, 51, 1-20. https://doi.org/10.1016/0040-1951(78)90048-3

[11] Ikeya, M. (2004) Earthquakes and Animals: From Folk Legends to Science. World Scientific, London, 295. https://doi.org/10.1142/5382

[12] Hjelmslev, L. (1988) Saggi linguistici. Edizioni Unicopli, Milano.

[13] Jakobson, R. (1966) Saggi di linguistica generale. Feltrinelli, Milano.

[14] Miller, P. (2018) Exerting Dominance over Your Dog Is the Wrong Way to Build a Good Relationship. Whole Dog Journal. https://www.whole-dog-journal.com/issues/14_12/features/Alpha-Dogs_20416-1.ht $\mathrm{ml}$

[15] John, W.S., Bradshaw, E.J., Blackwell, R. and Casey, A. (2009) Dominance in Domestic Dogs. Useful Construct or Bad Habit? Journal of Veterinary Behavior: Clinical Applications and Research, 4, 135-144. https://doi.org/10.1016/j.jveb.2008.08.004

[16] Grimm, D. (2015) How the Wolf became the Dog. Science, April, n. 348. https://www.sciencemag.org/news/2015/04/how-wolf-became-dog

[17] Rigutti, A. (2015) Atlante di anatomia. Giunti Editore, Firenze.

[18] Rigutti, A. (2015) Atlante di fisiologia. Giunti Editore, Firenze.

[19] Jones, S. (1992) The Cambridge Encyclopedia of Human Evolution. Cambridge University Press, Cambridge.

[20] Galignano, M. (2011) Pedagogia e scienza della voce. Presentazione di F. Fussi. Edizioni Omega, Torino.

[21] Zerneri, L. (2013) Manuale di foniatria, audio-foniatria e compendio di logopedia. Edizioni Omega, Torino.

[22] Drake, A.G. (2011) Despelling Dog Dogma: An Investigation of Heterochrony in Dogs using 3D Geometric Morphometric Analysis of Skull Shape. National Center for Biotechnology Information. National Library of Medicine. Evolution and Development, 13, 204-213. https://doi.org/10.1111/j.1525-142X.2011.00470.x

[23] Wayne, K.R. (1986) Cranial Morphology of Domestic and Wild Canids: The Influence of Development on Morphological Change. Evolution, 40, 243-261. https://doi.org/10.1111/j.1558-5646.1986.tb00467.x

[24] Mu, L. and Yang, S. (1990) Electromyographic Study on End-to-End Anastomosis of the Recurrent Laryngeal Nerve in Dogs. The Laryngoscope, 100, 1009-1017. https://doi.org/10.1288/00005537-199009000-00017

[25] Coppinger, R. and Coppinger, L. (2002) A New Understanding of Canine Origin, Behavior and Evolution. University of Chicago Press, Chicago.

[26] Coppinger, R. and Smith, C.K. (1983) The Domestication of Evolution. Environmental Conservation, 10, 283-292. https://doi.org/10.1017/S0376892900012984

[27] Coppinger, R. and Coppinger, L. (2001) Dogs: A Startling New Understanding of Canine Origin, Behavior, and Evolution. Scribner, New York.

[28] Compagnoni, R. and Sandrone, R. (1981) Lineamenti Geo-Petrografici Delle Alpi 
Cozie Italiane tra la Val di Susa e la Val Po. Federazione Piemontese di Mineralogia e Paleontologia, Torino.

[29] Lacidonia, G., Manuello, A., Carpinteri, A., Niccolini, G., Agosto A. and Durin, G. (2010) Acoustic and Electromagnetic Emissions in Rocks under Compression. Proceedings of SEM Annual Conference, Indianapolis, 7-10 June 2010, 57-64.

[30] Carpinteri, A., Lacidogna, G., Manuello, A. and Borla, O. (2009) Piezonuclear Transmutations in Brittle Rocks under Mechanical Loading: Microchemical Analysis and Geological Confirmations. In: Kounadis, A.N. and Doutos, E.E.G., Eds., Symposium on Recent Advances in Mechanics, Academy of Athens, Athens, Book Part III, 361-382.

[31] McCaig, M. (1977) Permanent Magnets in Theory and Practice. Pentech Press, London.

[32] Meiklejohn, W.H. and Bean, C.P. (1957) New Magnetic Anisotropy. Physical Review, 105, 904-913. https://doi.org/10.1103/PhysRev.105.904

[33] Aharoni, A. (1996) Introduction to the Theory of Ferromagnetism. Clarendon Press, New York.

[34] De Liso, G., Fidani, C. and Viotto, A. (2014) Multi-Parametric Monitoring System of Associated Seismic Phenomenology and Unusual Animal Behaviour in Western Piedmont. Advances in Research, 2, 303-319. https://doi.org/10.9734/AIR/2014/8873

[35] Caputo, M. (1987) Sismologia e Segnali Precursori dei Terremoti. Calderini Editore, 1987.

[36] Dobrovolsky, I.P., Zubkov, S.I. and Miachkin, V.I. (1979) Estimation of the Size of Earthquake Preparation Zones. Pure Application in Geophysics, 117, 1025-1044. https://doi.org/10.1007/BF00876083

[37] Ghosh, D. and Sengupta, D.R. (2009) Anomalous Radon Emission as Precursor of Earthquake. Journal of Applied Geophysics, 69, 67-81. https://doi.org/10.1016/j.jappgeo.2009.06.001

[38] Riggio, A., Santulin, M. and Tamaro, A. (2014) Prima analisi dei dati di Radon inseriti nella banca dati del Progetto S3. Atti del Convegno GNGTS 2014, 25-27 Novembre, Bologna.

[39] Vaupotic, J., Riggio, A., Santulin, M., Zmazek B. and Kobal, I. (2010) A Radon Anomaly in Soil Gas at Cazzaso, NE Italy, as a Precursor of an ML $=5.1$ Earthquake. Nukleonika, 55, 507-511.

[40] Volpe, P.E. (2006) Determinazione di radon e radio nelle acque della Valle Pellice e di Susa. Società Geologica Italiana, 3, 33-36.

[41] Hart, V., Nováková, P., Malkemper, E., Begall, S. and Hanzal, V.R. (2013) Dogs Are Sensitive to Small Variations of the Earth's Magnetic Field. Frontiers in Zoology, 10, 80. https://doi.org/10.1186/1742-9994-10-80

[42] Nießner, C., Denzau, S., Malkemper, E.P., Gross, J.C., Burda, H., Winklhofer, M. and Peichl L. (2016) Cryptochromel in Retinal Cone Photoreceptors Suggests a Novel Functional Role in Mammals. Scientific Reports, 6, Article Number: 21848. https://doi.org/10.1038/srep21848

[43] Fidani, C., Albarello, D., Arcaleni, M., Martinelli, G. and Siciliani, P.M. (2012) Emilia Earthquake: VLF Transmitters and ELF Signal from the Central Italy Electromagnetic Network (CIEN). Atti 2 del $31^{\circ}$ Convegno Nazionale GNGTS, Po-tenza, 20-22 November 2012, 415-420.

[44] De Liso, G. and Viotto A. (2014) Rumbles and Mistpoeffers in Val Pellice (Western 
Piedmont) with and without Earthquakes: A Comparison between Radon 222 and Infra-Sounds Monitoring and Unusual Animal Behaviors. Atti del Convegno GNGTS, 25-27 Novembre 2014, Bologna.

[45] di Torre Pellice, C., di Torino, P. and Regolatore Generale, P. (1996) Analisi delle previsioni urbanistiche Indagini Geomorfologiche (Circolare P.G.R. n.7/LAP 8 maggio 1996).

[46] Municipality of Torre Pellice (2008) Geological Map. ARPA of the Piedmont Region.

[47] Rikitake, T. (1968) Earthquake Prediction. Earth Science Review, 4, 245-282. https://doi.org/10.1016/0012-8252(68)90154-2

[48] Rikitake, T. (1987) Previsione dei Terremoti. Dario Flaccovio Editore, 1987, 301. Palermo.

[49] Rikitake, T. (1982) Earthquake Prediction Work in Sichuan Province, China, with Special Reference to the Song-pan-Pingwu Earthquakes F. T. Wu, Ed., Earthquake Research in China. Chinese Geophysics, 2, 139-156.

[50] Woith, H., Peyersen, G.M., Hainzel, S. and Dahm, T. (2018) Can Animals Predict Earthquakes? Bulletin of the Seismological Society of America, 108, 1031-1045. https://doi.org/10.1785/0120170313 